\title{
Statusreproduktion und Mobilitätseffekte beruflicher Weiterbildung* Status Reproduction and the Effects of Further Training on Mobility
}

\section{Rolf Becker}

Universität Bern, Institut für Erziehungswissenschaft, Abteilung Bildungssoziologie, Fabrikstrasse 8, CH-3012 Bern, Switzerland, rolf.becker@edu.unibe.ch

\begin{abstract}
Klaus Schömann
Deutsches Institut für Erwachsenenbildung, Leibniz-Zentrum für Lebenslanges Lernen e.V., Heinemannstrasse 12-14, D-53175 Bonn, Germany, schoemann@die-bonn.de
\end{abstract}

Zusammenfassung: Es wird für Westdeutschland untersucht, ob sowohl der intergenerationale Abstieg als auch der intergenerationale Statuserhalt Beweggründe für berufliche Weiterbildung sind. Des Weiteren wird untersucht, ob mit beruflicher Weiterbildung ein intergenerationaler Abstieg ausgeglichen oder das Risiko für intergenerationale Abwärtsmobilität minimiert werden kann. Mit Längsschnittdaten der Deutschen Lebensverlaufsstudie finden sich für westdeutsche Frauen und Männer in fünf aufeinander folgenden Geburtskohorten im Zeitraum von 1949 bis 1999 Indizien dafür, dass das Statuserhaltmotiv ein Weiterbildungsmotiv für Personen sein könnte, die einen intergenerationalen Statusverlust erfahren haben. Mit Weiterbildungsanstrengungen können im weiteren Berufsverlauf intergenerationale Abstiege ausgeglichen werden. Ferner haben statuskonsistente Teilnehmer geringere Risiken für einen intergenerationalen Abstieg als Nichtteilnehmer. Berufliche Weiterbildung hat statusreproduzierende Wirkungen im Berufsverlauf.

Schlagworte: Berufliche Bildung; Berufliche Weiterbildung; Intergenerationale Mobilität; Berufsverlauf; Statuserhalt; Statusreproduktion; Lebensverlauf.

\begin{abstract}
Summary: Based on a life course perspective, this paper explores whether intergenerational status decline initiates employees' participation in further vocational training. Additionally, it investigates whether further training compensates for status decline across generations and whether it contributes to decreasing the risk of such decline in the course of an occupational career. Results based on the German Life History Study of West German women and men from several birth cohorts observed between 1949 and 1999 indicate that status maintenance may be an important motivation for individuals who have experienced intergenerational status decline. By means of further training, these persons are able to compensate for downward mobility. Status consistent participants who undertake further training are more likely to be able to reduce the risk of intergenerational status decline than non-participants. Further vocational training supports status maintenance across generations.
\end{abstract}

Keywords: Vocational Training; Further Training; Intergenerational Mobility; Occupational Career; Status Maintenance; Status Reproduction; Life Course.

\section{Einleitung}

Werden die Teilnahme an beruflicher Weiterbildung und deren Konsequenzen für den Berufsverlauf betrachtet, dann kristallisieren sich wenige individuelle Gründe dafür heraus, warum sich Erwerbstätige beruflich weiterbilden (Becker \& Hecken 2011: 376 ff.). So bilden sie sich weiter, um ihre beruflichen Fertigkeiten zu erhalten, berufliche Kennt-

\footnotetext{
* Für hilfreiche Hinweise und Kommentare danken wir den anonymen Gutachtern und den Herausgebern sowie Thomas Bühler, Karl Ulrich Mayer und Walter Müller. Den Beitrag widmen wir unserem Doktorvater und Mentor Prof. Dr. Karl Ulrich Mayer zu seinem 70. Geburtstag.
}

nisse aufzufrischen, vorhandene Qualifikationen weiterzuentwickeln, berufliche Produktivität zu steigern sowie sich an neue qualifikatorische Anforderungen auf dem Arbeitsmarkt und in betrieblichen Arbeitsabläufen anzupassen. Sie verfolgen hiermit im Wesentlichen das Ziel, ihre Erwerbsund Einkommenschancen zumindest zu wahren oder gar zu verbessern - sofern es für sie keine anderen Möglichkeiten gibt, dieses Ziel zu erreichen (Schömann \& Becker 1995; Müller \& Jacob 2008). Berufliche Weiterbildung dient somit hauptsächlich der Einkommensoptimierung (Becker \& Schömann 1996; Wolter \& Schiener 2009) und dem Erhalt des (beruflichen) Status (Becker 1991; Beicht \& Walden 2006). 
Dass der intragenerationale Statuserhalt im Berufsverlauf, etwa das Vermeiden von Einkommensverlusten, beruflichen Abstiegen oder Arbeitslosigkeit, ein zentraler Grund neben anderen - wie etwa dem Streben nach höherem Einkommen, mehr Arbeitsmarktflexibilität oder einer Beförderung - für berufliche Weiterbildung sein kann, ist in der Arbeitsmarktforschung empirisch belegt (Becker \& Hecken 2011: 395 ff.). Diese Fakten korrespondieren mit dem Bestreben von Menschen, Statusverluste zu vermeiden (Ormel et al. 1999). Zu diesem Zwecke, so die Theorie sozialer Produktionsfunktion, investieren sie (kontinuierlich) in Bildung, weil in modernen Gesellschaften mit marktorientierter Wirtschaftsordnung und wohlfahrtsstaatlichen Institutionen die Bildung die multifunktionale Ressource ist, die den Sozialstatus bestimmt, den sie bereits vom Elternhaus kennen. Empirisch ungeklärt ist jedoch die Rolle von beruflicher Weiterbildung für den intergenerationalen Statuserhalt. $\mathrm{Ob}$ sich Personen beruflich weiterbilden, um zumindest den sozialen Status zu reproduzieren, den ihre Eltern bereits erreicht haben, ist für Deutschland weder systematisch und zeitlich-kontinuierlich für den Berufsverlauf noch im Kohortendesign für einen langen historischen Zeitraum untersucht worden.

Dies ist insofern erstaunlich, als in der gegenwärtigen soziologischen Bildungsforschung insbesondere das Statuserhaltmotiv angeführt wird, um zu erklären, wie Eltern in die Bildung ihrer Kinder investieren. Demnach verfolgen Eltern bei der Schul- und Berufsausbildung ihrer Kinder das Ziel, dass ihre Kinder mindestens die Klassenlage - und damit dieselbe soziale Anerkennung (Prestige) sowie den gleichen materiellen Wohlstand (Einkommen) - erreichen, die sie bereits erlangt haben (Stocké 2010). Des Weiteren liefern Studien Hinweise dafür, dass dieses Statuserhaltmotiv von den Kindern übernommen wird, wenn es um Bildungs- und Berufsentscheidungen in ihrem späteren Lebenslauf geht etwa zwischen Berufsausbildung und Allgemeinbildung am Ende der Pflichtschulzeit (Glauser 2015). Ebenso zeigen beispielsweise Becker \& Hecken (2007) sowie Schindler \& Reimer (2010: 629), dass das Statuserhaltmotiv auch für Studienberechtigte die Entscheidung für oder gegen ein Hochschulstudium (Reimer \& Pollak 2010) oder die Studienfachwahl (Becker et al. 2010) bestimmt. Inwieweit das Statuserhaltmotiv bei der intergenerationalen Berufsvererbung und für den Zugang zu Statuspositionen eine Rolle spielt, ist empirisch ungeklärt (Wegener 1989: 271; Stocké 2010: 84).
Der in der Generationenabfolge angestrebte Statuserhalt wird jedoch von den Kindern beim Berufseinstieg im Anschluss an ihre Ausbildung oder im späteren Berufsverlauf - etwa bemessen am beruflichen Prestige (Blossfeld 1989) oder an der Klassenlage (Mayer \& Carroll 1987) - trotz aller vorherigen Bemühungen in der formalen Erstausbildung nicht immer realisiert (Mayer \& Blossfeld 1990). Bei einem intergenerationalen Abstieg könnten, so die zentrale Annahme in diesem Beitrag, abwärts mobile Erwerbstätigen die berufliche Weiterbildung als ein probates Mittel unter mehreren für nachholende intergenerationale Statusreproduktion ansehen, wenn sie - trotz aller vorherigen Bildungsund Karriereanstrengungen - (noch) nicht den Herkunftsstatus erreicht haben.

$\mathrm{Ob}$ sich intergenerational abgestiegene Personen tatsächlich weiterbilden und ob mit beruflicher Weiterbildung dann ein intergenerationaler Statusverlust kompensiert werden kann, soll in der vorliegenden Studie empirisch geklärt werden. Ebenso wird untersucht, ob statuskonsistente Personen, die bereits zumindest die gleiche Klassenlage wie ihre Eltern erreicht haben, Investitionen in die berufliche Weiterbildung nutzen können, um Risiken für etwaige intergenerationale Abstiege im weiteren Berufsverlauf zu minimieren.

Der Beitrag ist wie folgt aufgebaut: Im nächsten Abschnitt werden neben einem knappen Überblick über den Forschungsstand theoretische Annahmen über den Zusammenhang von Statuserhalt und beruflicher Weiterbildung dargestellt, aus denen empirisch überprüfbare Hypothesen abgeleitet werden. Der dritte Abschnitt umfasst die Beschreibung der Datenbasis, Variablen und Analyseverfahren, der vierte die Darstellung der empirischen Befunde. Eine Zusammenfassung der Ergebnisse und Schlussfolgerungen folgen im abschließenden fünften Abschnitt.

\section{Theoretischer Hintergrund}

\subsection{Statusreproduktion und berufliche Weiterbildung - der Forschungsstand}

Dass das Statuserhaltmotiv einen Anreiz für entsprechendes Bildungsverhalten darstellt, ist weder für die Schul- und Berufsausbildung oder das Universitätsstudium noch für die Teilhabe an beruflicher Weiterbildung unumstritten (Jacob \& Weiss 2011; Van de Werfhorst \& Hofstede 2007). In der empirischen Forschung sind die Befunde zur Rolle des Statuserhalts für Bildungsentscheidungen durch- 
aus disparat. Beispielsweise schlussfolgert Stocké (2010: 84), dass Studien, die den Statuserhalt am Erreichen des elterlichen Bildungsniveaus festmachen, deswegen nicht zu eindeutigen Befunden kommen, weil mit dem Statuserhaltmotiv das Erreichen des elterlichen Berufsstatus gemeint sein könnte. Diese Sicht ist vor dem Hintergrund der intergenerationalen Klassenreproduktion plausibel und berücksichtigt auch, dass im Zuge der Bildungsexpansion das elterliche Bildungsniveau in der Generation der Kinder nicht mehr für die Reproduktion des elterlichen Berufsstatus hinreichend ist. Van de Werfhorst \& Hofstede (2007) haben ebenso wie Breen \& Yaish (2006) mangels adäquater Messung der subjektiv erwarteten Eignung von Bildungsgängen zur Vermeidung eines intergenerationalen Statusabstiegs keine eindeutigen Belege für das Statuserhaltmotiv liefern können. Stocké (2007) hingegen hat die zwischen den Sozialschichten variierende Rolle des Statuserhaltmotivs für Bildungsentscheidungen am Ende der Grundschulzeit direkt nachweisen können. Glauser (2015) gelingt dies für die Entscheidung für eine Berufsausbildung oder für eine fortgesetzte Allgemeinbildung nach der Pflichtschulzeit. Becker \& Hecken (2007) wiederum haben hierfür indirekte Belege bei der Wahl zwischen Studium und Berufsausbildung und Becker et al. (2010) für die Studienfachwahl zwecks Statusreproduktion vorgelegt. Gabay-Egozi et al. (2009) dagegen zeigen für 12-jährige Schulkinder in Tel Aviv (Israel) anhand einer den späteren Universitätszugang vorstrukturierenden Entscheidung zwischen unterschiedlichen Fächern in der Sekundarstufe I, dass das (direkt gemessene) Statuserhaltmotiv irrelevant ist. Jacob \& Weiss (2011) haben für die USA den Wiedereinstieg in die formale höhere Bildung nach vorheriger Erwerbstätigkeit und die Einflüsse des sozialen Hintergrunds - insbesondere des Statuserhalts - für diesen Prozess untersucht. Bei einem indirekten Test des Statuserhaltmotivs und Strebens nach nachholender Statusreproduktion finden Jacob \& Weiss (2011) keine eindeutigen Zusammenhänge für Statuserhaltmotiv und Weiterbildungsanstrengungen. Gründe hierfür können zum einen die Schlussfolgerung aus einer indirekten Hypothesenüberprüfung sein, ohne die theoretisch angenommenen Mechanismen direkt gemessen zu haben. Zum anderen kann die Rückkehr in die höhere Schulbildung nach dem Berufseinstieg ein Spezialfall sein, dessen Untersuchung zu anderen Ergebnissen führt, als wenn mehrere Wechsel zwischen Bildungs- und Beschäftigungssystem, andere Übergänge in die berufliche Weiterbildung und schließlich Berufsverläufe länger betrachtet werden (Becker 1991: 352).

Diese disparaten Befunde sind jedoch aus methodischen und theoretischen Gründen kein ausreichender Beleg dafür, dass das Statuserhaltmotiv bei intergenerational abgestiegenen oder vom intergenerationalen Abstieg bedrohten Erwerbstätigen kein gewichtiger Grund ist, in die eigene berufliche Weiterbildung zu investieren. Wenn Kinder in der Sozialisation im Elternhaus durch das elterliche Statuserhaltmotiv geprägt und der Bildungs- und Berufsverlauf durch die darauf basierenden (elterlichen) Bildungsentscheidungen (Stocké 2010) sowie durch elterliche Aspirationen gesteuerte Berufswahlen (Glauser 2015) strukturiert werden, ist nicht ausgeschlossen, dass neben den Freunden und Kollegen (Schindler et al. 2011) das Elternhaus weiterhin die Referenzkategorie für die Beurteilung der eigenen Mobilität und Klassenlage ist. Diese Vermutungen werden durch das, Wisconsin Model of Status Attainment gestützt. Es geht davon aus, dass die intergenerationale Bildungs- und Berufsreproduktion größtenteils über Einflüsse der Bezugsgruppen (Haller \& Portes 1973: 71) und über Bildungs- und Berufsaspirationen von Eltern und ihren Kindern vermittelt wird (Sewell et al. 1969). Dass der anhand einer Statusskala gemessene Effekt der sozialen Herkunft immer positiv und in der Regel statistisch signifikant für den Status des Einstiegsberufs ist (Mayer \& Blossfeld 1990), kann durchaus als Folge des Statuserhaltmotivs interpretiert werden, das in die Bildungs- und Berufsaspirationen einfließt. Dass dann der spätere soziale oder berufliche Status immer weniger direkt vom Status des Elternhauses abhängt, während der von der sozialen Herkunft abhängige Status beim Berufseinstieg in hohem Maße den Status im Berufsverlauf beeinflusst, sind weitere Indizien dafür, dass auch im späteren Lebensverlauf das Motiv für den intergenerationalen Statuserhalt bedeutsam für die berufliche Karriere ist. Demnach dürfte die Klassenlage des Elternhauses ein entscheidender Referenzpunkt für die Beurteilung der Klassenlage der Kinder in späteren Lebensverlaufsphasen darstellen. An die ,Status Position Theory“ (Keller \& Zavalloni 1964) und ,Prospect Theory“ (Kahneman \& Tversky 1979) anknüpfend, kann vermutet werden, dass das Statuserhaltmotiv nachhaltig wirkt und dass Kinder soziale Abstiege zu vermeiden versuchen. Sollten selbst bei entsprechenden Bildungserfolgen statuserhaltende berufliche Positionen im Berufsverlauf nicht erreicht werden, könnte berufliche Weiterbildung subjektiv als ein weiterer Erfolg versprechender Weg angesehen werden, der über 
die Förderung beruflicher Mobilität dazu beiträgt, doch noch den Status zu erreichen, welcher der Klassenposition des Elternhauses entspricht.

\subsection{Brückenhypothesen über die soziale Selektivität von beruflicher Weiterbildung}

Warum aber bilden sich nicht alle Erwerbstätigen weiter, um den Statuserhalt abzusichern oder - im Falle eines vorherigen intergenerationalen Statusverlustes - den Status zu reproduzieren? Obgleich sozialer Status ein erstrebenswertes Gut ist, besteht nicht für jedes Individuum zwingend die Notwendigkeit, in berufliche Weiterbildung zu investieren, um den bereits erreichten Status abzusichern (Backes-Gellner et al. 2007). In einer Vielzahl von Fällen dürften formale Erstausbildung sowie stetiges Lernen im Arbeitsprozess ausreichend sein, um den Statuserhalt in der Generationenabfolge langfristig garantieren zu können (Offerhaus et al. 2010). Erst wenn dieser z. B. wegen suboptimaler Ausbildung oder Karriere noch nicht erreicht ist oder z. B. wegen drohender Arbeitslosigkeit gefährdet zu sein scheint, wird oftmals in berufliche Weiterbildung investiert (Büchel \& Pannenberg 2004). In dem Fall kann sie als individuelle Investition in die intergenerationale Statusreproduktion aufgefasst werden.

Dieser Fall ist nicht für alle Beschäftigtengruppen gleichermaßen gegeben. Denn wenn die bislang vom Elternhaus erreichte Klassenlage die Referenzposition in der gesellschaftlichen Hierarchie darstellt, von dem aus Statusverluste bewertet werden (Jæger \& Holm 2012), dann variieren zwischen den sozialen Klassen die Anreize für berufliche Weiterbildung zwecks Statuserhalt. Beispielsweise haben unqualifizierte Arbeiter und einfache Angestellte, also soziale Klassen am unteren Ende der gesellschaftlichen Stratifikation, kein oder nur ein geringfügiges Risiko für Statusverluste (abgesehen von $\mathrm{Ar}$ beitslosigkeit) und daher auch kein besonderes Interesse an beruflicher Weiterbildung zwecks Statuserhalts. Oftmals fehlen ihnen auch entsprechende Mittel und Gelegenheiten dafür, da sie zumeist in sekundären Arbeitsmärkten mit geringen Weiterbildungschancen beschäftigt sind. Für Individuen aus den Mittelschichten und der oberen Dienstklasse hingegen kann der Erhalt des sozialen Status in der Generationenabfolge ein zentrales Motiv sein, sich für mehr oder weniger kontinuierliche Weiterbildung zu entscheiden, falls ein Statusverlust droht oder bereits eingetreten ist. Folglich dürfte aus Sicht dieser statushöheren Individuen die berufliche Weiterbildung ein strategisch bedeutsames Mittel sein, um den Status zu erhalten oder bei einem vorherigen Statusabstieg diesen wiederherstellen zu wollen.

Dass die Chance für berufliche Weiterbildung nicht immer unter der Kontrolle einer erwerbstätigen Person selbst steht, gehört zum grundlegenden Forschungsstand (Becker \& Hecken 2011). Humankapital-, signal- und segmentationstheoretische Ansätze verweisen auf die Fremdselektion beim Zugang zur beruflichen Weiterbildung, die sich für gering qualifizierte Arbeiter und Angestellte nachteilig auswirkt. So weist eine Vielzahl von Studien darauf hin, dass Beschäftigte in internen Arbeitsmärkten - etwa in großen privatwirtschaftlichen Betrieben und im öffentlichen Dienst - oder in gehobener beruflicher Stellung (z. B. leitende Angestellte) oder in Beschäftigungsverhältnissen mit institutionalisierter Weiterbildung (z. B. Beamte) oder auf Arbeitsplätzen mit hohen Qualifikationsanforderungen (z. B. Akademiker) besonders günstige Chancen haben, sich beruflich weiterbilden zu können (Becker 1991). Die Chance, sich weiterbilden zu können, ist auch für diejenigen Erwerbspersonen gering, bei denen Arbeitgeber oder Weiterbildungsträger annehmen, dass eine erfolgreiche Teilnahme und die damit einhergehende Produktivität ungewiss sind (Schömann \& Becker 1995). Bei dem hinreichend oft belegten Zusammenhang von sozialer Herkunft, Leistungsfähigkeit und subjektiver Erwartung, eine Ausbildung erfolgreich abzuschließen, dürften sich potentielle Weiterbildungsteilnehmer aus den unteren Sozialschichten folglich im Nachteil befinden. Dementsprechend sind sie in Fort- und Weiterbildungsmaßnahmen unterrepräsentiert.

Neben selektiven Opportunitäten der Arbeitsmärkte, der Beschäftigungsverhältnisse und der Qualifikationsanforderungen beruflicher Tätigkeiten (Schindler et al. 2011) können auch individuelle Restriktionen (etwa subjektiv erwartete Kosten und Nutzen einer Weiterbildung) Personen davon abhalten, sich weiterzubilden. ${ }^{1}$ Für Personen aus den

\footnotetext{
${ }^{1}$ Weil die Renditen der beruflichen Weiterbildung unsicher sind, erscheinen Investitionen in die Weiterbildung mit beruflichem Aufstieg als Ziel für Personen mit geringen Ressourcen und Erfolgswahrscheinlichkeiten als riskant und aufwendig. Denn hierbei tritt das Problem auf, „dass die Kosten der Maßnahme in der Regel sofort anfallen und zu einer unmittelbaren finanziellen Belastung führen, während der Nutzen zunächst ungewiss ist und, wenn überhaupt, erst nach und nach realisiert werden kann" (Bardeleben et al. 1994: 10). Nach Bardeleben et al. (1994: 16) sahen mehr als ein Drittel der westdeutschen Teilnehmer und fast ein Viertel der ostdeutschen Teilnehmer ihre erwarteten Nutzen infolge einer beruflichen
} 
unteren Sozialschichten erscheinen aufgrund geringerer Erwerbseinkommen und Erfolgsaussichten zusätzliche Investitionen in berufliche Weiterbildung sehr viel aufwendiger und riskanter als für beruflich erfolgreiche Personen aus höheren Sozialschichten. Selbst wenn die sozial und beruflich Privilegierten in der Anfangsphase ihres Berufsverlaufs einen intergenerationalen Abstieg hinnehmen mussten, weisen sie vermutlich - bei gleichen subjektiven Erwartungen von Weiterbildungsrenditen (z. B. Einkommen, Arbeitsplatzsicherheit, Aufstiegsmöglichkeiten) - aufgrund des Statuserhaltmotivs eine höhere Weiterbildungsmotivation auf als aus niedrigen Sozialschichten stammende Erwerbstätige. Wie bereits erwähnt, sind deren subjektiv erwartete Aussichten, erfolgreich in die berufliche Weiterbildung zu investieren und diese gewinnbringend für einen nachholenden Statusaufstieg oder für einen dauerhaften Statuserhalt im Berufsverlauf einzusetzen, vergleichsweise höher. Selbst wenn diese Erfolgsaussichten gering erscheinen, verfügen Statusgruppen aus vorteilhaften Klassenlagen über andere Ressourcen und Wege als die berufliche Weiterbildung, um nachträglich den Statuserhalt sicherzustellen und einem Statusverlust vorzubeugen. Hierzu zählen neben sozialen Netzwerken (Granovetter 1974) auch für die Statusreproduktion vorteilhafte Gelegenheitsstrukturen in ihren typischen Beschäftigungsbereichen (Offerhaus et al. 2010).

\subsection{Hypothesen}

Ausgehend von den theoretischen Überlegungen werden folgende Hypothesen abgeleitet und empirisch überprüft: 1.) Zwecks Statusreproduktion bilden sich erwerbstätige Personen mit intergenerationalem Statusabstieg eher beruflich weiter als Personen ohne intergenerationale Abwärtsmobilität (Hypothese des Statuserhaltmotivs). 2.) Bei vorherigem intergenerationalem Abstieg können Teilnehmer an beruflicher Weiterbildung den Statusverlust eher ausgleichen als Nichtteilnehmer (Hypothese der Kompensation eines Statusverlusts). 3.) Statuskonsistente Personen - d. h. Personen, die sich in der gleichen oder einer günstigeren Klassenlage als ihr Elternhaus befinden - können mittels beruflicher Weiterbildung eher ihre Klassenlage erhalten als Nichtteilnehmer (Hypothese des nachhaltigen Statuserhalts). 4.) Sowohl für den Zugang zu beruflicher Fort- und Weiterbildung als auch für die Verwertung der Wei-

Weiterbildung als erfüllt an, wobei jedoch keine Informationen dazu vorliegen, ob Nichtteilnehmer ihre Nichtteilnahme bedauert haben. terbildung für die intergenerationale Statusreproduktion sind Teilnehmer aus den höheren Sozialschichten generell im Vorteil (Hypothese der persistenten Ungleichheit nach sozialer Herkunft).

\section{Datenbasis und Variablen}

\subsection{Datenbasis}

Um diese Hypothesen überprüfen zu können, bedarf es neben einer strukturell-individualistischen Lebensverlaufsperspektive auch ereignisorientierter Längsschnittdaten über Bildungs-, Berufs- und Weiterbildungsverläufe von Frauen und Männern in unterschiedlichen Geburtsjahrgängen (Blossfeld \& Huinink 2001). Nur mit solchen, längere Zeitspannen des Erwerbslebens lückenlos abdeckenden Daten kann in angemessener Weise ein kausaler Zusammenhang von Weiterbildung und sozialer Mobilität aufgezeigt werden. Befragungen im Querschnitt (z. B. Mikrozensus) oder Retrospektivdaten für kurze Zeiträume (12 Monate wie im Berichtssystem Weiterbildung oder Adult Education Survey 2012) sind für die hier verfolgte Fragestellung methodisch ungeeignet (Becker 1991: 357).

Ereignisorientierte Verlaufsdaten der Deutschen Lebensverlaufsstudie (GLHS) erfüllen diese Anforderungen für Westdeutschland für die Jahre von 1949 bis 1999 (Mayer 2008). Sie werden daher für die empirische Analyse verwendet. ${ }^{2}$ In der GLHS wurden Frauen und Männer in unterschiedlichen Geburtsjahrgängen befragt. Die folgenden Analysen beschränken sich auf die Daten der westdeutschen Lebensverlaufsstudie, die Informationen über in Deutschland geborene Personen aus den Kohorten 1929-31, 1939-41, 1949-51, 1964 und 1971 enthält. ${ }^{3}$ Die zwischen 1981 und 1983 erhobenen Kohorten der um 1930, 1940 und 1950 Geborenen be-

\footnotetext{
2 Die verwendeten Daten sind bei GESIS (https://dbk.gesis. org/dbksearch/GDESC2.asp?no=0033\&DB=D) archiviert und können dort bezogen werden: 1) ZA2645: Lebensverläufe und gesellschaftlicher Wandel: Lebensverläufe und Wohlfahrtsentwicklung (Westdeutschland, Kohorten 1929-31, 1939-41, 1949-51): http://dx.doi.org/10. 4232/1.2645; 2) ZA3927: Ausbildungs- und Berufsverläufe der Geburtskohorten 1964 und 1971 in Westdeutschland (Kohorten 1964 und 1971): http://dx.doi.org/10. 4232/1.3927.

${ }^{3}$ Die Kohorten 1959-61 wurden wegen fehlender Zeitangaben zum Beginn einer beruflichen Weiterbildung nicht berücksichtigt. Die 1919-21 Geborenen bleiben unberücksichtigt, weil ihre Arbeitsmarkteintritte vor der Gründung der Bundesrepublik Deutschland erfolgten.
} 
stehen aus 2171 Befragten (Brückner \& Mayer 1998). Ferner wurden zwischen 1998 und 1999 in Zusammenarbeit mit dem Institut für Arbeitsmarkt- und Berufsforschung (IAB) die Lebensverlaufsdaten von 2909 Frauen und Männern der Jahrgänge 1964 und 1971 erhoben (Hillmert 2004). Die Daten der westdeutschen Teilstudien haben sich bereits bei früheren Analysen zur beruflichen Weiterbildung (Böhnke 1997; Schömann \& Becker 1995) und zu ihren Wirkungen auf den Berufs- und Einkommensverlauf bewährt (Becker 1991; Becker \& Schömann 1996). Im Folgenden werden nur Personen ab dem Alter von 15 Jahren und für den Zeitraum nach Gründung der Bundesrepublik Deutschland ab 1949 untersucht.

Die Angaben der Befragten sind Retrospektivdaten (Mayer 2008). Sie wurden gebeten, mit möglichst exakten Zeitangaben ihren Lebensverlauf in verschiedenen Bereichen wie etwa sozialer Herkunft, Schul- und Berufsausbildung, Berufsverlauf und beruflicher Weiterbildung zu rekonstruieren. Hochgradig institutionalisierte Ereignisse und Zustände wie etwa Heirat und Ehe werden bei solch einem Verfahren in der Regel zuverlässig erinnert, während bei weniger institutionalisierten Ereignissen und Zuständen wie etwa Zeitpunkt und Dauer der beruflichen Weiterbildung systematische Erinnerungsfehler umso wahrscheinlicher sind, je weiter sie in der Zeit zurückliegen (Reimer 2003). Solche Fehler sind wahrscheinlich, da Weiterbildungen vermutlich punktuelle Ereignisse im Lebensverlauf sind. Das dürfte die Identifikation einzelner Weiterbildungen im Berufsverlauf ebenso erschwert haben wie ihre Abgrenzung zur schulischen und beruflichen Ausbildung. Insbesondere ergaben sich für die älteren Kohorten Probleme bei der Datenaufbereitung, weil die Befragten in der Retrospektive die einzelnen Sequenzen der beruflichen Weiterbildung nicht mehr exakt erinnern oder voneinander abgrenzen konnten (Becker 1991: 356). Dennoch bürgen sorgfältige Vorbereitung und Erhebung der Daten (Matthes et al. 2007) sowie intensive Nachbefragungen, systematische Recherchen und Edition der Daten für eine hohe Datenqualität (Brückner \& Mayer 1998; Hillmert 2004; Mayer 2008).

\subsection{Abhängige und unabhängige Variablen ${ }^{4}$}

Die Analyseeinheiten sind Arbeitsplatzepisoden im Berufsverlauf der befragten Personen. Die erste abhängige Variable ist die Teilnahme an beruflicher

\footnotetext{
${ }^{4}$ Die Verteilungen der Variablen sind in Tabelle A1 im Online-Anhang (www.zfs-online.org) dokumentiert.
}

Weiterbildung innerhalb einer Arbeitsplatzepisode. Herangezogen werden die von den Befragten genannten - in der Hauptsache non-formalen - beruflichen Weiterbildungen (Eisermann et al. 2014: 479). ${ }^{5}$ Das Fort- und Weiterbildungsvolumen wird aufgrund vieler fehlender Werte und Informationen ebenso wenig berücksichtigt wie die Art der Weiterbildung. Insofern besteht diesbezüglich das Problem unbeobachteter Heterogenität, weil die Wirksamkeit beruflicher Weiterbildung auch deutlich zwischen den einzelnen Typen der Weiterbildungen differieren dürfte. Hierfür wären ebenso weiterführende Untersuchungen notwendig wie detaillierte Angaben zu vorherigen Weiterbildungsteilnahmen.

Ein weiteres Problem ergibt sich für die Datenanalyse und Interpretation der Befunde, dass aufgrund fehlender Informationen zu den Motiven für die berufliche Weiterbildung auch nicht beurteilt werden kann, ob sich die Teilnehmer zum Zwecke des Statuserhalts weiterbilden. Deswegen muss der subjektive Sinn des Weiterbildungsverhaltens - das Statuserhaltmotiv - indirekt theoriegeleitet aus dem Weiterbildungsverhalten unter Kontrolle der Ausgangslage (hier: des intergenerationalen Abstiegs) geschlossen werden. Solche indirekten Schlussfolgerungen aufgrund fehlender direkter Messung von Handlungszielen und sozialen Mechanismen sind in der empirischen Sozialforschung sehr häufig (Brüderl 2004). Jedoch haben sie wie im vorliegenden Fall, in dem das Statuserhaltmotiv als gegeben angenommen wird, den Nachteil, dass aus unzutreffenden Annahmen empirisch zutreffende Phäno-

\footnotetext{
${ }^{5}$ Die Originalfrage lautet folgendermaßen: „Haben Sie irgendwann einmal Kurse oder Veranstaltungen besucht, die Ihrer beruflichen Weiterbildung dienten? Es spielt dabei keine Rolle, ob es sich um Tages- oder Wochenendseminare gehandelt hat oder um Kurse von längerer Dauer und ob die Initiative dazu vom Betrieb, vom Arbeitsamt oder von Ihnen selbst ausging“. Die Befragten sollten auch angeben, um welchen Kurs bzw. welche Veranstaltung es sich dabei gehandelt hat (z. B. Fachrichtung, Lehrgangsthema). Aber die Angaben konnten nicht - auch nicht in Kombination mit der Angabe, ob es sich um einen Kurs bzw. Lehrgang gehandelt hat - für alle Kohorten valide nach dem Typus der Weiterbildung (z. B. Anpassungsoder Aufstiegsweiterbildung, etc.) eingeordnet werden. Ebenso konnte wegen fehlender Fragen für die älteren Kohorten nicht eindeutig zwischen inner- und über- bzw. außerbetrieblicher Weiterbildung unterschieden werden. Schließlich wird die informelle Weiterbildung (,on-the-job training', selbstgesteuertes Lernen oder Besuch von Fachtagungen oder -messen) nicht berücksichtigt, da sie trotz ihres Bedeutungszuwachses nicht zur beruflichen Weiterbildung im engeren Sinne zählt (Büchel \& Pannenberg 2004: 76).
} 
mene vorhergesagt werden können. Insofern müssten unsere Analysen und Befunde, die wie andere Forschungsergebnisse als vorläufige zu betrachten sind, mit Daten repliziert werden, die valide Informationen zum Statuserhaltmotiv der Befragten beinhalten.

Die zweite abhängige Variable ist die intergenerationale Mobilität zwischen zwei Arbeitsplatzepisoden. Es wird zwischen intergenerationalen Aufund Abstiegen unterschieden. Bei den Analysen für die Weiterbildungsteilnahme liegt ein Statusverlust vor, wenn sich eine Person in der $n$-ten Arbeitsplatzepisode in einer niedrigeren Klassenlage als das Elternhaus befindet. Die Referenzkategorie sind statuskonsistente Personen. Diese haben in der $n$-ten Arbeitsplatzepisode die mindestens gleiche Klassenlage wie ihr Elternhaus.

Bei den Auswirkungen der Weiterbildung auf die intergenerationale Mobilität liegt ein (kompensatorischer) intergenerationaler Aufstieg dann vor, wenn eine Person - unter der Voraussetzung, dass sie in der jeweils beobachteten $n$-ten Arbeitsplatzepisode eine niedrigere Statusposition einnimmt als das Elternhaus (d.h. intergenerational abgestiegen ist) - bei einem Arbeitsplatzwechsel (d.h. beim Übergang vom $n$-ten zum $n+1$-ten Arbeitsplatz) in der darauffolgenden Arbeitsplatzepisode mindestens die Klassenlage des Elternhauses erreicht. Dieser Mobilitätsprozess wird lediglich für erwerbstätige Personen analysiert, welche in der $n$-ten Arbeitsplatzepisode eine niedrigere Klassenlage als ihre Eltern innehatten - also vor der Weiterbildung einen intergenerationalen Abstieg erfahren hatten. Zum anderen wird für statuskonsistente Personen die Wahrscheinlichkeit für intergenerationale Statusabstiege in der $n+1$-ten Arbeitsplatzepisode geschätzt, die bislang mindestens die gleiche Klassenlage wie ihre Eltern innehatten (d.h. zuvor nicht abgestiegen sind). Insgesamt wurden bei den Weiterbildungseffekten (operationalisiert als DummyVariablen: $1=\mathrm{ja} / 0=$ nein) nur die jeweiligen Risikomengen berücksichtigt, welche zuvor das komplementäre Ereignis nicht erfahren haben. In vorherigen Modellierungen ergaben sich enorme Schätzprobleme, wenn Beschäftigtengruppen einbezogen wurden, die in Bezug auf den Ausgangszustand und die abhängige Variable nicht zur Risikomenge gehörten. Effekte der sozialen Herkunft und aktuellen Klassenlage waren nicht interpretierbar.

Als eine weitere unabhängige Variable wird die intragenerationale Mobilität über den Wechsel der Klassenlage bei Tätigkeitswechsel berücksichtigt. Hierbei wird der Abstieg in der Klassenstruktur bei einem Wechsel von der vorherigen zur jeweils aktu- ellen (d. h. von der $n-1$-ten zur $n$-ten) Arbeitsplatzepisode gemessen. In der weiterführenden Analyse wird diese Variable mit einem intergenerationalen Abstieg beim Berufseinstieg kombiniert. Beides wird jeweils über eine Dummy-Variable kontrolliert (1=ja / $0=$ nein).

Die Klassenlage des Elternhauses und die Klassenlage der Erwerbstätigen im Berufsverlauf wird anhand des ,German Employment Class Schema (GEC) von Mayer und Aisenbrey (2007: 132) gemessen. Für die Klassenlage des Elternhauses wurde - für den Zeitpunkt, als die Befragten 15 Jahre alt waren - die höchste berufliche Stellung eines Elternteils herangezogen. Fehlende Angaben zur beruflichen Stellung des Vaters bzw. Stiefvaters wurden ersetzt durch die Angabe der (Stief-)Mutter. Der methodische Vorbehalt, die Klassenlage des Elternhauses könne zu irreführenden Ergebnissen führen, weil es sich um eine Messung zum Zeitpunkt handelt, als die Befragten 15 Jahre alt waren, und daher die Klassenmobilität des Elternhauses zu späteren Zeitpunkten nicht berücksichtigt wird, sodass die elterliche Klassenlage nicht mehr die adäquate Referenzposition darstellt, scheint zunächst plausibel. Jedoch müssten bei Eltern in älteren Kohorten und für deren spätere Lebenslaufphasen enorm hohe Mobilitätsraten angenommen werden. Dem stehen jedoch anders lautende Befunde über hohe Immobilität entgegen (Müller \& Pollak 2004). Für den Status der Kinder, also der Befragten, wurde jeweils die berufliche Stellung in einer der Arbeitsplatzepisoden berücksichtigt. Im vorliegenden Fall wird sie verwendet, da Weiterbildungschancen nach beruflicher Stellung verteilt sind. Dieses Klassenschema ist für unsere Fragestellung geeignet, weil es Besonderheiten der Beschäftigungsverhältnisse und des Staates als Arbeitgeber in Deutschland berücksichtigt. Hierbei wird, dem Vorschlag von Mayer \& Aisenbrey (2007) folgend, zwischen hierarchisch angeordneten Klassen - Arbeiterklasse, untere Mittelschicht, mittlere Mittelschicht und obere Dienstklasse - unterschieden. ${ }^{6}$

\footnotetext{
${ }^{6}$ Die Arbeiterklasse umfasst mithelfende Familienangehörige, Landwirte mit Land unter 10 Hektar, un- und angelernte Arbeiter, Angestellte mit einfacher Tätigkeit (z.B. Verkaufsberufe) und Beamte im einfachen Dienst. Die untere Mittelschicht schließt Facharbeiter, Vorarbeiter, Selbständige mit maximal einem Mitarbeiter sowie Landwirte mit einem Bodenbesitz unter 50 Hektar ein. Der mittleren Mittelschicht gehören Handwerks- und Industriemeister, Angestellte mit qualifizierter Tätigkeit (z. B. Sachbearbeiter), Beamte im mittleren Dienst, Selbständige mit bis zu 9 Angestellten sowie Landwirte mit mehr als 50 Hektar Land an. In der oberen Dienstklasse befinden sich Ange-
} 
Neben der Kohortenzugehörigkeit (Dummy-Variablen mit Jahrgang 1929-31 als Referenzkategorie) werden als weitere Zeitdimensionen die Berufserfahrung der Personen (in Monaten) und die historische Periode berücksichtigt. Die Periode wird wegen der Konfundierung der Zeitdimensionen mit gleichem Maßstab (Mayer \& Huinink 1990) - als Proxy für wirtschaftliche Rahmenbedingungen anhand der vorjährigen Arbeitslosenquote zu Beginn einer Arbeitsplatzepisode identifiziert. Der Wandel ökonomischer Kontexte sollte zugleich weitere Anreize für berufliche Weiterbildung darstellen (Schömann \& Becker 1995).

Für jede Arbeitsplatzepisode im Berufs- und Weiterbildungsverlauf wurden das Geschlecht der Befragten (Dummy-Variable: Frauen als Referenzkategorie) sowie deren Schul- und Berufsausbildung (Dummy-Variablen: maximal Hauptschulabschluss und keine Berufsausbildung als Referenzkategorie) als Indikatoren für Mechanismen und Prozesse der Selbst- und Fremdselektion in die Weiterbildung kontrolliert. Als Arbeitsplatz- bzw. Arbeitsmarktmerkmal wurden neben der Arbeitszeit (in Stunden) die Beschäftigung im öffentlichen Dienst (DummyVariable mit Privatwirtschaft als Referenzkategorie) und die Betriebsgröße (Anzahl der Beschäftigten in der Arbeitsstätte) als betriebliche und institutionelle Gelegenheitsstrukturen für Weiterbildung in Rechnung gestellt. Schließlich wurden Merkmale des vorherigen Berufsverlaufs („Vorgeschichte“) - wie Betriebs- oder Tätigkeitswechsel (Dummy-Variablen: 1=ja / $0=$ nein) - berücksichtigt, um die Kontingenz des Berufsverlaufs zu kontrollieren (Blossfeld 1989).

\subsection{Statistisches Verfahren und das Selektivitätsproblem}

Bezüglich der Effekte beruflicher Weiterbildung ist das sogenannte Selektivitätsproblem zu berücksichtigen. Es tritt hier auf, weil anstelle von in einem experimentellen Design erhobene Daten Umfragedaten verwendet werden, die eine zufällige Aufteilung von Versuchs- und Kontrollgruppe und Kontrolle der Weiterbildungsteilnahme als, treatment nicht zulassen (Morgan \& Winship 2007). Aufgrund der Selbst- oder Fremdselektion in beide

stellte mit hochqualifizierter Tätigkeit oder Leitungsfunktion (z. B. Prokurist), Angestellte mit Führungsaufgaben (z. B. Geschäftsführer), Beamter im gehobenen oder höheren Dienst (bzw. Richter), akademische bzw. freie Berufe (z. B. Arzt mit eigener Praxis, Rechtsanwalt) sowie Selbständige mit mindestens 10 Angestellten.
Gruppen der Teilnehmer und Nichtteilnehmer ist es im quasi-experimentellen Design nicht ohne weiteres möglich, kausale Effekte beruflicher Weiterbildung aufzudecken (Becker \& Schömann 1996). So kann der Weiterbildungseffekt nicht durch einen Vergleich beider Gruppen ermittelt werden, indem beispielsweise in multivariaten Analysen die Teilnahme bzw. Nichtteilnahme durch eine DummyVariable indiziert wird. Denn wegen des, selectivity bias' ist der erwartete durchschnittliche Mobilitätseffekt beruflicher Weiterbildung nicht identisch mit der Differenz der Ergebnisvariablen für Teilnahme und Nichtteilnahme (Winship \& Sobel 2004). Die Indikation durch eine Dummy-Variable ist folglich keine brauchbare Lösung des Evaluationsproblems, weil sie zu verzerrten Schätzergebnissen führt.

Um dieses methodische Problem auf ein geringes Maß zu reduzieren, wird - in Anlehnung an Heckman (1979) - in bereits bewährter Weise vorgegangen (Becker \& Schömann 1996). ${ }^{7}$ Im ersten Schritt wird mittels einer Cox-Regression, eines semi-parametrischen Verfahrens der Ereignisanalyse, die soziale Selektivität der Weiterbildungsteilnahme geschätzt (Blossfeld \& Rohwer 1995). ${ }^{8}$ Die Neigung einer Person, sich weiterzubilden, wird zunächst als Hazardrate abgespeichert. Sie berücksichtigt hierbei sowohl die zeitliche Reihenfolge von Kausaleffekten (z. B. Statusabstieg als Beweggrund vor Weiterbildung als Konsequenz) als auch die Kausalität als stochastischen statt deterministischen Prozess (z. B. Wahrscheinlichkeit einer Weiterbildung infolge eines Statusverlusts). Der Begründung von Blossfeld \& Rohwer (1997) folgend wird die Hazardrate als Chance eines Individuums verstanden, zu handeln. Sie wird mit der tatsächlichen Weiter-

\footnotetext{
${ }^{7}$ Möglicherweise werden bei ereignisorientierten Verlaufsdaten sowohl das Selektivitätsproblem als auch das fundamentale Problem der kausalen Inferenz überschätzt. Zum einen können ein und dieselben Personen über einen längeren Zeitraum betrachtet werden, und zwar zugleich - über die Zeit verteilt - in den Zuständen der Teilnahme oder Nichtteilnahme an beruflicher Weiterbildung (Blossfeld \& Rohwer 1997). Zum anderen gilt dies auch für die Wirkung von beruflicher Weiterbildung, da ein und dieselben Personen sowohl im Zustand der Statuskonsistenz (Reproduktion) als auch in der Statusinkonsistenz (Abstieg) beobachtet werden (Pötter \& Blossfeld 2001).

${ }^{8}$ Dieses Verfahren wird hier deswegen verwendet, weil keine eindeutige theoriegeleitete Verteilungsannahme für die Hazardrate getroffen werden kann. Die Proportionalitätsannahme ist für Geschlecht und Kohorte gültig. Zudem hat sich das wegen der Produktivitäts- und Rentabilitätsannahme der Humankapitaltheorie naheliegende Gompertz-Makeham-Modell empirisch nicht bewährt (Schömann \& Becker 1995).
} 


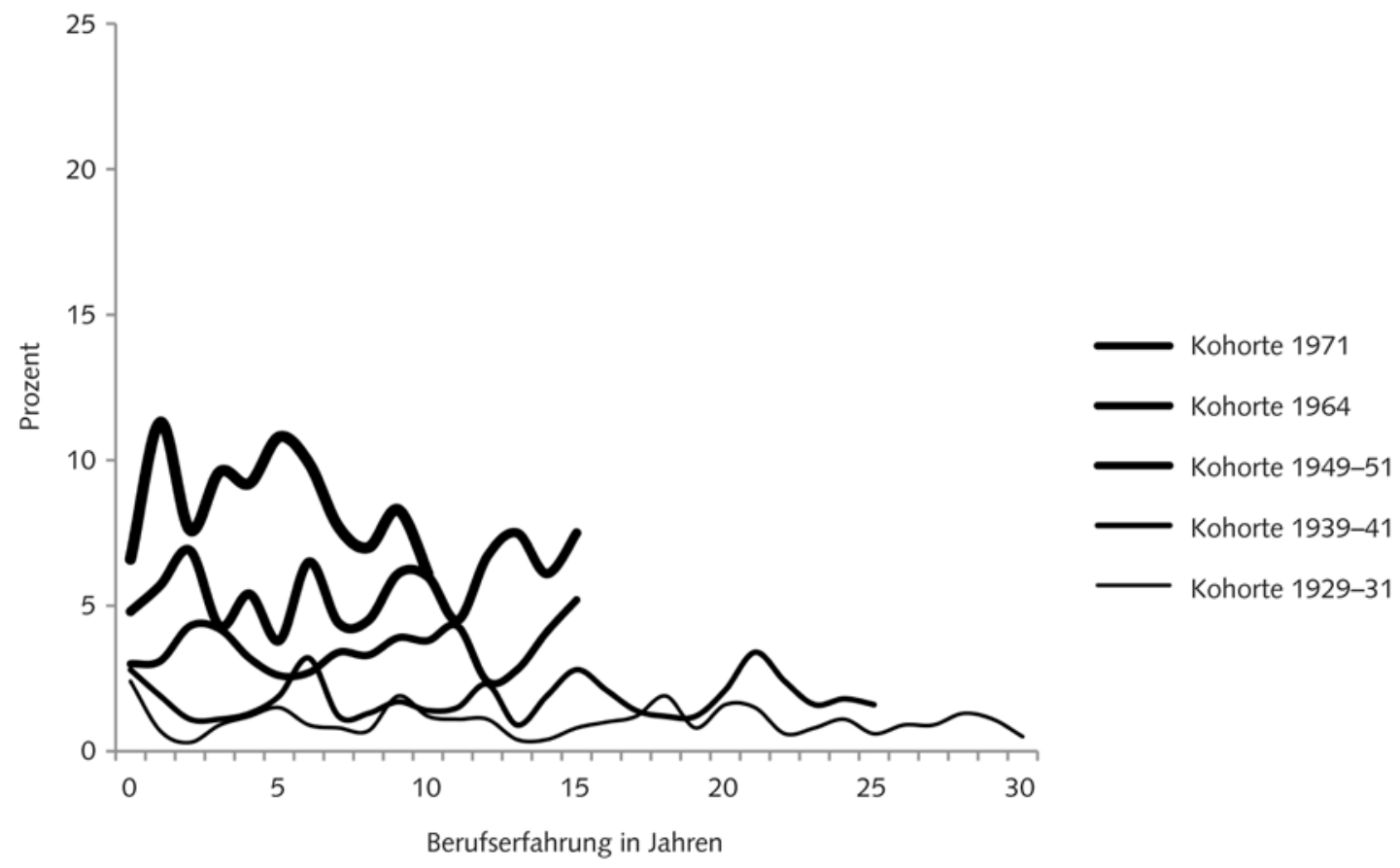

Datenbasis: GLHS - eigene Berechnungen

Abbildung 1 Eintritte erwerbstätiger Personen in die berufliche Weiterbildung (prozentuale Anteile an Erwerbstätigen)

bildungsteilnahme gewichtet. Im zweiten Schritt, der eigentlichen Analyse der Mobilitätsprozesse, geht diese gewichtete Hazardrate als probabilistischer Indikator $P$ (Teilnahme) für eine vorherige Weiterbildungsteilnahme in die Schätzung von Weiterbildungseffekten mittels der Cox-Regression ein.

Es besteht Einigkeit darin, dass die Lösung für das ,selection bias problem" außerhalb der formalen Statistik liegt. Weil der Prozess der Selektion eher ein theoretisches als ein statistisches Problem ist, kommt es darauf an, die Selektivität theoriegeleitet zu modellieren. Hierbei ergibt sich für unsere Analyse ein Dilemma, auf das bereits Heckman (1979) hingewiesen hat. Wenn der Prozess der sozialen Selektivität beim Zugang zur beruflichen Weiterbildung theoriegeleitet modelliert werden soll und wenn für die Effekte der Weiterbildung auf die intergenerationale Mobilität die gleichen Mechanismen angenommen werden, dann tritt das Problem der Multikollinearität auf, falls ein und dieselben Variablen verwendet werden. Daher wird in der folgenden Analyse die soziale Selektivität der Weiterbildung möglichst mit den Variablen modelliert, die für die Mobilitätsanalysen nicht zwingend benötigt werden.
Solch eine zentrale Variable ist die Zugehörigkeit $z u$ einem Geburtsjahrgang. Zum einen ist für die soziale Mobilität in Westdeutschland eine markante Kohortendifferenzierung empirisch belegt (Mayer \& Solga 1994), zum anderen ist für Erwerbstätige in Westdeutschland die Kohortendifferenzierung bei der beruflichen Weiterbildung ebenfalls mehrfach nachgewiesen worden (Becker 1991: 358; Böhnke 1997; Becker \& Hecken 2011: 387, 390). Werden Eintritte in die berufliche Weiterbildung nach Berufserfahrung betrachtet, so unterscheiden sich die Jahrgänge systematisch danach, dass die Eintrittsraten von Kohorte zu Kohorte über den gesamten Berufsverlauf hinweg immer höher sein werden (Abbildung 1). ${ }^{9}$ Während - für den gesam-

\footnotetext{
${ }_{9}$ Alters- und periodenspezifische Weiterbildungsquoten, die typischerweise mit Querschnittsdaten berechnet werden (z. B. Kuwan \& Thebis 2005: 23, 34-35), führen in der Regel zu irreführenden Verteilungen in der Zeit, weil die Referenzkategorien für die Altersklassen oder historischen Zeitpunkte wechseln. Alleine die zeitliche Variation des Eintritts in die Erwerbstätigkeit nach der Ausbildung durch die unterschiedliche Verweildauer in unterschiedlichen Bildungsgängen ist verantwortlich dafür, dass altersspezifische Weiterbildungsquoten für Erwerbstätige kaum aussagekräftig sind.
} 
Tabelle 1: Kohortenspezifische Eintritte in die berufliche Weiterbildung - nur Erwerbstätige in Westdeutschland, 1949-1999

\begin{tabular}{|c|c|c|c|c|c|c|}
\hline \multirow[t]{2}{*}{ Modell } & \multicolumn{2}{|c|}{ Insgesamt (1) } & \multicolumn{2}{|c|}{ Frauen (2) } & \multicolumn{2}{|c|}{ Männer (3) } \\
\hline & $\boldsymbol{\beta}$ & S.E. & $\boldsymbol{\beta}$ & S.E. & $\boldsymbol{\beta}$ & S.E. \\
\hline Kohorte 1929-31 & Referenz & & Referenz & & Referenz & \\
\hline Kohorte 1939-41 & $0,426 * * *$ & 0,100 & $0,943 * * *$ & 0,211 & $0,264^{*}$ & 0,115 \\
\hline Kohorte 1949-51 & $1,080 * * *$ & 0,101 & $1,762 * * *$ & 0,204 & $0,798 * * *$ & 0,123 \\
\hline Kohorte 1964 & $1,625 * * *$ & 0,088 & $2,136 * * *$ & 0,192 & $1,477 * * *$ & 0,100 \\
\hline Kohorte 1971 & $2,113 * * *$ & 0,091 & $2,790 * * *$ & 0,194 & $1,833 * * *$ & 0,107 \\
\hline Pseudo-R ${ }^{2}$ (McFadden) & \multicolumn{2}{|c|}{0,02} & \multicolumn{2}{|c|}{0,04} & \multicolumn{2}{|c|}{0,03} \\
\hline Episoden / Ereignisse & \multicolumn{2}{|c|}{$13.231 / 2.496$} & \multicolumn{2}{|c|}{$5.749 / 1.028$} & \multicolumn{2}{|c|}{$7.482 / 1.468$} \\
\hline
\end{tabular}

ten Berufsverlauf betrachtet - die Weiterbeteiligung für die älteste Kohorte der um 1930 Geborenen durchweg unter einem Prozent (gemessen an allen Erwerbstätigen zu den jeweiligen Berufsjahren) liegt, bewegt sie sich für die um 1940 Geborenen zwischen 2 und 3 Prozent. Die Weiterbildungsquoten für die Kohorte 1949-51 liegen im Schnitt bei 3 bis 4 Prozent. Bei den jüngeren Kohorten steigen sie auf Werte zwischen 4 und 7 Prozent (Jahrgang 1964) und für die Kohorte 1971 bewegen sie sich im Intervall von 7 bis 11 Prozent. In der Logik des Berufsverlaufs sind keine eindeutigen Muster, aber kohortenspezifische Intervalle für die Eintrittsraten in eine berufliche Weiterbildung auszumachen. So gibt es - bei konstanten Unterschieden zwischen den Kohorten - weiterhin Indizien dafür, dass die meisten Weiterbildungsaktivitäten in den ersten 5 Jahren und dann - für die nach dem Zweiten Weltkrieg Geborenen - verstärkt wieder nach 10 Jahren des Berufsverlaufs erfolgen und dann mehr oder weniger stagnieren. ${ }^{10}$

Diese Befunde werden durch multivariate Schätzungen untermauert (Tabelle 1). Für alle Erwerbstätigen sowie für Frauen und Männer getrennt ist ersichtlich, dass mit der Abfolge der Kohorten die Neigung, sich weiterzubilden, sukzessive ansteigt. Modell 1 wird herangezogen, um die soziale Selektivität für berufliche Weiterbildung durch die Kohortenzugehörigkeit zu kontrollieren. Im vorliegenden Fall ist diese Vorgehensweise aus folgendem Grund vertretbar: Die Kohortendifferenzierung so-

\footnotetext{
10 Im Übrigen verweisen die stetig steigenden Weiterbildungsquoten darauf hin, dass es sich nicht um reine Retrospektivartefakte handeln kann, da die zeitliche Distanz von Befragungs- zu Weiterbildungszeitpunkt nicht mit dem Geburtsjahr korreliert.
}

wohl der intergenerationalen Mobilität als auch der beruflichen Weiterbildung geht einher mit der Bildungsexpansion und der sukzessiv gestiegenen Mobilität im Berufsverlauf, der Expansion des Staates als Arbeitgeber und der sukzessiv gestiegenen Frauenerwerbstätigkeit und schließlich mit der wirtschaftlichen Modernisierung und der stetig gewachsenen Notwendigkeit, sich permanent weiterzubilden. Die Kohortenzugehörigkeit als erklärende Variable für die Weiterbildung schließt somit diese Wandlungsprozesse und ihre Korrelate ein.

Dadurch wird bei den Regressionsanalysen für die Effekte beruflicher Weiterbildung eine endogene Beziehung zwischen erklärenden Variablen ausgeschlossen, indem der Geburtsjahrgang durch die kohortenspezifische Neigung, sich im Berufsverlauf weiterzubilden, ersetzt wird. Diese Größe steht zwar in engem Zusammenhang mit den erklärenden Variablen für die Neigung, sich weiterzubilden, stellt aber in diesem Fall keine Linearkombination anderer erklärender Variablen dar. Somit wird das Problem der Konfundierung und Multikollinearität zumindest abgeschwächt.

\section{Empirische Ergebnisse}

\subsection{Statusverlust und Teilnahme an beruflicher Weiterbildung}

Zunächst werden aus sozialstruktureller und arbeitsmarkttheoretischer Perspektive für alle $\mathrm{Er}$ werbstätigen die Strukturen ihrer Teilnahme an beruflicher Weiterbildung modelliert (Modell 1 in Tabelle 2). Wie bei allen früheren Analysen (auch mit einer höheren Zahl erklärender Variablen sowie der Hinzunahme formaler und mehrfacher Ausbil- 


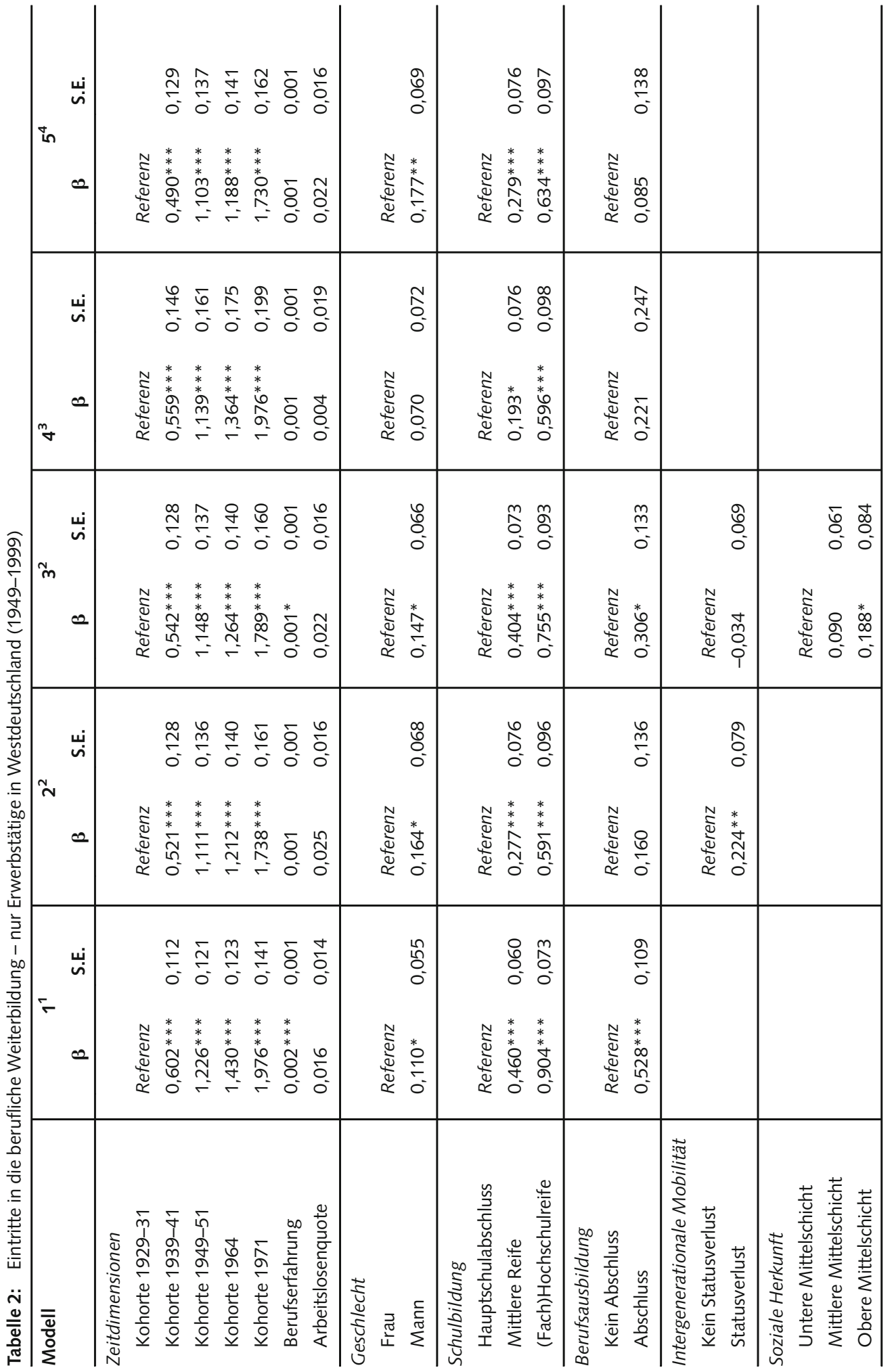




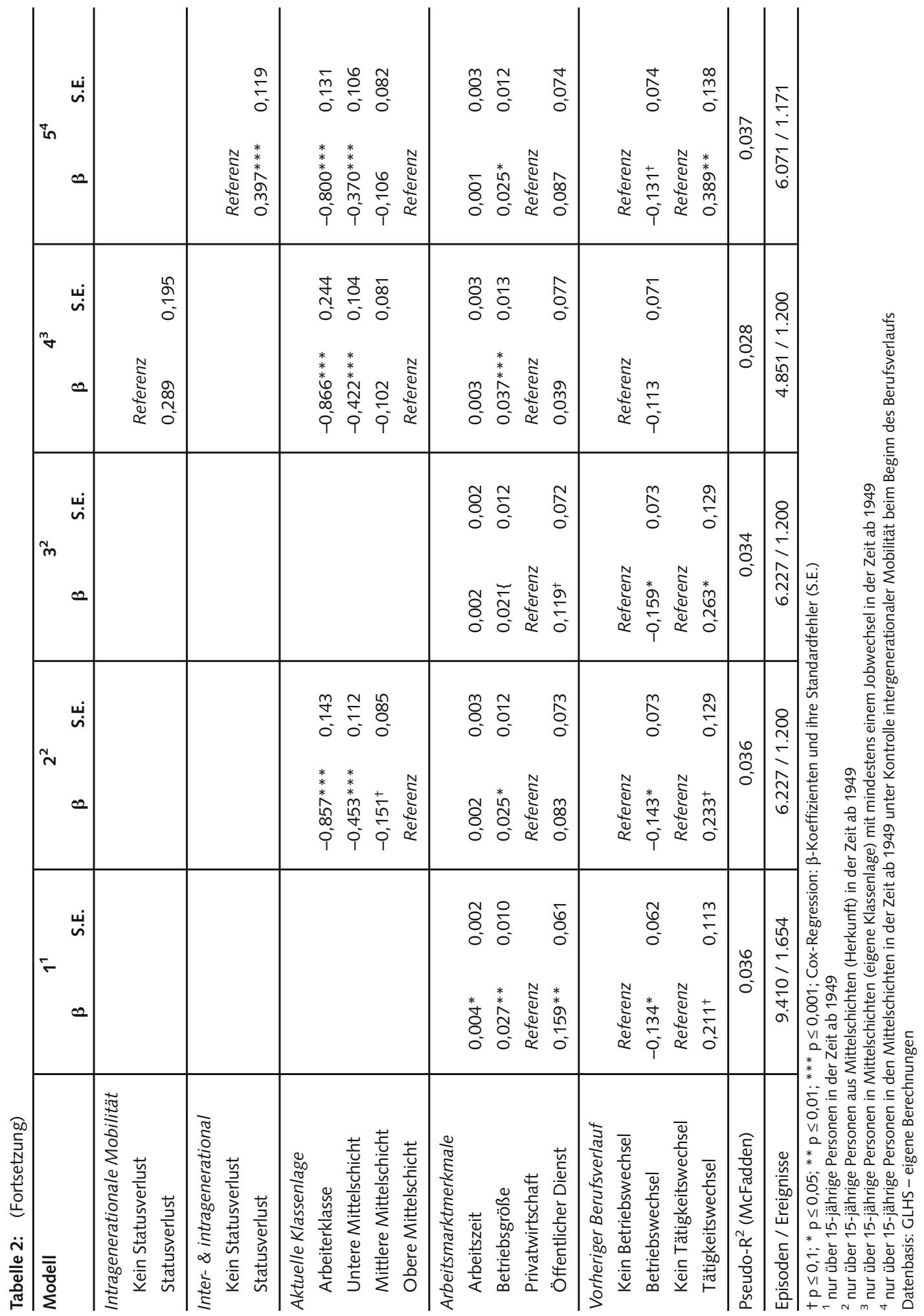


dung und von aggregierten Arbeitsmarktindikatoren aus der amtlichen Statistik) ist die statistische Erklärungskraft der Modellierungen relativ niedrig (vgl. z. B. Schömann \& Becker 1995).

Für die Neigung, sich weiterzubilden, besteht neben der bereits geschilderten Kohortendifferenzierung ein Zusammenhang mit der Berufserfahrung. Dieser Befund soll hier nicht weiterverfolgt werden, da die Berufsverläufe der einzelnen Kohorten unterschiedlich lang beobachtet wurden. Für Erwerbstätige gibt es offensichtlich keinen statistisch signifikanten Zusammenhang zwischen periodenspezifisch aggregierter Arbeitslosigkeit in der Erwerbsbevölkerung und Teilnahme an beruflicher Weiterbildung.

Männer bilden sich eher als Frauen weiter, und besser gebildete Erwerbstätige (höhere Schulbildung und abgeschlossene Berufsausbildung) weisen günstigere Chancen auf, sich weiterbilden zu können. Neben diesem „Matthäus-Effekt“ gibt es Effekte interner Arbeitsmärkte, wonach Erwerbstätige mit regulärer Arbeitszeit sowie Beschäftigung im öffentlichen Dienst und in großen Betrieben über günstigere Weiterbildungsmöglichkeiten verfügen. Personen mit vorherigem Wechsel ihrer beruflichen Tätigkeit bilden sich eher weiter, während Personen mit einem vorherigen Arbeitgeberwechsel eine geringere Chance oder Neigung für Weiterbildung haben.

Ausgehend von diesem Referenzmodell, werden jetzt ausschließlich für die aus allen Mittelschichten stammenden Frauen und Männer Zusammenhänge von intergenerationalem Statusabstieg und Eintritt in die berufliche Weiterbildung untersucht (Modell 2). Aus der Arbeiterklasse stammende Personen werden nicht berücksichtigt, da sie im Unterschied zu der aus der Mittelschicht stammenden Risikomenge per definitionem keinen intergenerationalen Statusabstieg erfahren können. Zunächst bilden sich - unter den aus den Mittelschichten stammenden Erwerbstätigen - die Personen eher weiter, die zuvor intergenerational abgestiegen sind, als die Personen, die nicht abwärts mobil waren. Der intergenerationale Statusverlust ist offensichtlich ein Anreiz für berufliche Weiterbildung. Ein Abstieg in die Arbeiterklasse oder in die untere Mittelschicht bringt allerdings geringere Chancen, sich weiterbilden zu können, mit sich, da Arbeitern und einfachen Angestellten geringere Weiterbildungschancen gewährt werden.

Wird statt der erreichten Klassenlage die soziale Herkunft kontrolliert, dann gibt es keinen separaten signifikanten Effekt des intergenerationalen Statusabstiegs für den Eintritt in die berufliche Weiterbildung (Modell 3). Hier zeigt sich, dass sich
Erwerbstätige aus der oberen Dienstklasse statistisch signifikant eher weiterbilden als Personen aus den anderen Mittelschichten. Auch dieser Befund kann als Hinweis dafür gelten, dass sie möglicherweise wegen des Statuserhalts kontinuierlich in Weiterbildung investieren.

Werden nur die erwerbstätigen Personen in den Mittelschichten mit mindestens zwei Tätigkeitsepisoden betrachtet, die folglich einem Risiko für einen intragenerationalen Abstieg unterliegen können, dann ist festzustellen, dass berufliche Abstiege, die gleichzeitig einen Abstieg in der Klassenstruktur einer Gesellschaft (ohne Referenz zur sozialen Herkunft) bedeuten, nicht zu zusätzlichen Weiterbildungsanstrengungen führen (Modell 4). Somit hat eher die intergenerationale als die intragenerationale Mobilität einen Einfluss auf das Weiterbildungsverhalten im Berufsverlauf.

Diese Vermutung kann erhärtet werden, wenn wiederum nur die Erwerbstätigen in den Mittelschichten als Risikomenge herangezogen werden, die bereits beim Beginn ibres Berufsverlaufs einen intergenerationalen Statusabstieg erfahren haben (Modell 5). Hier wird diese Risikomenge betrachtet, weil frühere Analysen ergeben haben, dass gerade beim Berufseintritt intergenerational abgestiegene Personen besondere Mobilitätsanstrengungen an den Tag legen (Becker 1994). Sind sie beim Berufseinstieg intergenerational abgestiegen, dann bilden sie sich - bei Kontrolle der aktuellen, im Berufsverlauf erreichten Klassenlage und der intragenerationalen Mobilität im weiteren Berufsverlauf eher weiter als statuskonsistente Personen.

Die bisherigen Befunde sprechen dafür, dass intergenerationale Statusabstiege eher zu Weiterbildungsanstrengungen (möglicherweise zwecks nachholender Statusreproduktion) führen als intragenerationale Klassenmobilität im Berufsverlauf (d. h. berufliche Karriere). Damit wird die Hypothese des Statuserhaltmotivs über Investitionen in berufliche Weiterbildung zumindest indirekt empirisch gestützt. Folglich beschränkt sich die Analyse von Weiterbildungseffekten sowohl auf Prozesse der intergenerationalen Statusreproduktion bei statusinkonsistenten Personen als auch auf Prozesse des Statuserhalts bei statuskonsistenten Personen.

\subsection{Mobilitäts- und Statuseffekte beruflicher Weiterbildung bei vorherigen Klassenabstiegen}

Nunmehr werden Mobilitäts- und Statuseffekte beruflicher Weiterbildung ausschließlich für intergenerational abgestiegene Erwerbstätige untersucht. 
Erwerbstätige, die über die berufliche Karriere in die obere Dienstklasse gelangt sind, werden naturgemäß ausgeschlossen. In der Tat scheint für intergenerational abgestiegene Personen die berufliche Weiterbildung ein probates Mittel zu sein, um einen intergenerationalen Statusverlust auszugleichen (Tabelle 3).

Allerdings ist hier wiederum darauf hinzuweisen, dass die statistische Erklärungskraft der Modelle relativ gering ist, was zum einen auf die geringe Zahl der Ereignisse für diese spezielle Risikomenge zurückgeführt werden kann. Zum anderen ist nicht ausgeschlossen, dass die Wirkung beruflicher Wei- terbildung für die Kompensation intergenerationaler Statusabstiege im Vergleich zu nicht kontrollierten Einflussfaktoren oder zu anderen Ereignissen wie etwa Steigerung des Erwerbseinkommens relativ gering ist.

Bei Kontrolle des Geschlechts, der Berufserfahrung, der Arbeitsmarktentwicklung und der im Berufsverlauf erreichten Klassenlage können erwerbstätige Personen über Weiterbildung nachholend in die Klassenlage aufsteigen, welche ihrer sozialen Herkunft entspricht, und somit die Klassenlage des Elternhauses reproduzieren (Modell 1). Offensichtlich sind statusinkonsistente Frauen gegenüber Män-

Tabelle 3: Effekte beruflicher Weiterbildung auf intergenerationale Mobilität in Westdeutschland (1949-1999): Wahrscheinlichkeit für intergenerationalen Statusaufstieg nach vorheriger intergenerationaler Abwärtsmobilität ${ }^{1}$

\begin{tabular}{|c|c|c|c|c|c|c|}
\hline \multirow[t]{2}{*}{ Modell } & \multicolumn{2}{|c|}{1} & \multicolumn{2}{|c|}{2} & \multicolumn{2}{|c|}{3} \\
\hline & $\boldsymbol{\beta}$ & S.E. & $\boldsymbol{\beta}$ & S.E. & $\boldsymbol{\beta}$ & S.E. \\
\hline \multicolumn{7}{|l|}{ Geschlecht } \\
\hline Frau & Referenz & & Referenz & & Referenz & \\
\hline Mann & $0,734 * * *$ & 0,099 & $0,623 * * *$ & 0,100 & $0,674 * * *$ & 0,104 \\
\hline \multicolumn{7}{|l|}{ Zeitdimensionen } \\
\hline Berufserfahrung & $-0,005^{* * *}$ & 0,001 & $-0,004 * * *$ & 0,001 & $-0,004^{* * *}$ & 0,001 \\
\hline Arbeitslosenquote & 0,014 & 0,013 & 0,000 & 0,013 & 0,000 & 0,014 \\
\hline \multicolumn{7}{|l|}{ Aktuelle Klassenlage } \\
\hline Arbeiterklasse & 0,102 & 0,126 & $0,743 * * *$ & 0,142 & $0,742 * * *$ & 0,146 \\
\hline Untere Mittelschicht & $-0,314^{*}$ & 0,157 & 0,154 & 0,168 & 0,158 & 0,173 \\
\hline Mittlere Mittelschicht & Referenz & & Referenz & & Referenz & \\
\hline \multicolumn{7}{|l|}{ Berufliche Weiterbildung } \\
\hline P(Teilnahme) & $1,665 * * *$ & 0,422 & $1,631 * * *$ & 0,441 & $1,753 * * *$ & 0,463 \\
\hline \multicolumn{7}{|l|}{ Schulbildung } \\
\hline Hauptschulabschluss & & & Referenz & & Referenz & \\
\hline Mittlere Reife & & & $0,466 * * *$ & 0,110 & $0,515 * * *$ & 0,113 \\
\hline (Fach)Hochschulreife & & & $1,248 * * *$ & 0,158 & $1,398 * * *$ & 0,164 \\
\hline \multicolumn{7}{|l|}{ Berufsausbildung } \\
\hline Kein Abschluss & & & Referenz & & Referenz & \\
\hline Abschluss & & & $1,043^{* * *}$ & 0,169 & $1,001 * * *$ & 0,172 \\
\hline \multicolumn{7}{|l|}{ Arbeitsmarktcharakteristik } \\
\hline Betriebsgröße & & & & & $-0,018$ & 0,016 \\
\hline Privatwirtschaft & & & & & Referenz & \\
\hline Öffentlicher Dienst & & & & & $-0,029$ & 0,131 \\
\hline Pseudo-R ${ }^{2}$ (McFadden) & 0,0 & & 0,0 & & 0,0 & \\
\hline Episoden / Ereignisse & 3.274 & 504 & 3.274 & 504 & 3.091 & 480 \\
\hline
\end{tabular}

\footnotetext{
${ }^{1}$ Cox-Regression - nur über 15-jährige Personen in der Zeit ab 1949 ohne Personen in der oberen Mittelschicht

${ }^{*} p \leq 0,05 ;{ }^{*} p \leq 0,01 ; * * * p \leq 0,001$

Datenbasis: GLHS - eigene Berechnungen
} 
nern im Nachteil, wenn es um die Kompensation eines intergenerationalen Abstiegs geht, der mit zunehmender Berufserfahrung immer unwahrscheinlicher wird. In der beobachteten Periode - zumindest bis Ende der 1980er Jahre - könnte die Prägung durch das ,male breadwinner model ' dazu geführt haben, dass Frauen stärker als Männer über den Status des Partners gesellschaftlich verortet wurden.

Ferner ist zu berücksichtigen, dass statusinkonsistente Personen unabhängig von beruflicher Weiterbildung über Investitionen in die formale Erstausbildung Statusabstiege kompensieren können. Höhere Schulabschlüsse und erfolgreiche Berufsausbildungen können mobilisiert werden, um über berufliche Mobilität den Statuserhalt im Generationenzusammenhang wiederherzustellen (Modell 2). Die Einbettung in diverse Arbeitsmärkte hingegen trägt nicht signifikant zur Erklärung eines Statusaufstiegs zuvor abgestiegener Personen bei (Modell 3).

\subsection{Mobilitäts- und Statuseffekte beruflicher Weiterbildung bei Statuskonsistenz}

Abschließend wird untersucht, ob statuskonsistente Erwerbstätige - also Personen, die bislang zu unterschiedlichen Zeitpunkten in ihrem Berufsverlauf mindestens die gleiche Klassenlage wie ihre Eltern erreicht haben - Investitionen in die berufliche Weiterbildung nutzen können, um mögliche intergenerationale Statusverluste zu vermeiden. Intergenerationale Abstiege sind seltene Ereignisse im Berufsverlauf statuskonsistenter Frauen und Männer. Dieses Phänomen rührt von den qualifikatorischen Mobilitätsbarrieren in der Klassenstruktur Westdeutschlands her. Schul- und Berufsbildung und auch die Berufsmäßigkeit der Erwerbstätigkeit wirkten schon immer statuskonservierend. Aus einer Arbeiterfamilie stammende Personen sind von dieser Analyse aufgrund ihrer Herkunft ausgenommen.

In der Tat können statuskonsistente Personen mittels beruflicher Weiterbildung das Risiko für einen intergenerationalen Statusabstieg im Berufsverlauf minimieren (Modell 1 in Tabelle 4). So erleiden Weiterbildungsteilnehmer - bei Kontrolle von Geschlecht, Berufserfahrung, Arbeitsmarktentwicklung und aktueller, im Berufsverlauf erreichter Klassenlage - statistisch signifikant seltener intergenerationale Statusverluste als Nichtteilnehmer. Allerdings sind diese Befunde angesichts der geringen Effizienz der Schätzungen infolge äußerst gerin- ger Weiterbildungsbeteiligung der Abgestiegenen unter den statusinkonsistenten Personen (vgl. Tabelle 2) mit Vorbehalt zu sehen. Sozial privilegierte Männer unterliegen einem geringeren Risiko für intergenerationale Statusabstiege als negativ privilegierte Männer. Allerdings sind diese Befunde zu relativieren, wenn die erworbene Erstausbildung kontrolliert wird (Modell 2). Dieser Weiterbildungseffekt wird immer schwächer, werden weitere Merkmale der beruflichen Mobilität und Allokation kontrolliert (Modell 3).

Jedoch ist - unabhängig von vorherigen Weiterbildungen - die formale Erstausbildung in der Regel ausreichend oder zumindest hinreichend nützlich, um im Berufsverlauf intergenerationale Abstiege zu vermeiden. Neben der erfolgreichen Berufsausbildung, die allerdings einen schwachen Effekt aufzeigt, trifft dies nur für die Studienberechtigung zu. Mit einer niedrigeren Schulbildung gelingt dann der intergenerationale Statuserhalt in einem deutlich geringeren Maße (Modell 2). Auch hier liegt ein Matthäus-Effekt vor, indem die Erwerbstätigen mit vorteilhafter Schul- und Berufsausbildung weitere Statusvorteile haben, wenn sie - und hier sind sie, wie bereits gesehen, im Vorteil - zusätzlich in berufliche Weiterbildung investieren (können).

Der ohnehin schwache Effekt der Berufsausbildung ist nicht mehr gegeben, wenn Arbeitsmarktmerkmale kontrolliert werden (Modell 3). Wie zu erwarten, sind Beschäftigte in internen Arbeitsmärkten vor allem im öffentlichen Dienst und in großen Betrieben Beschäftigte - vor intergenerationalen Abstiegen geschützt. Das sind wiederum Beschäftigungsbereiche, in die gut ausgebildete Absolventen des Bildungssystems eher gelangen als weniger gut ausgebildete Arbeitskräfte. Diese Kontexte bieten für die dort Beschäftigten vorteilhafte Gelegenheiten, die intergenerationale Statusreproduktion und damit den Statuserhalt in der Generationenabfolge dauerhaft sicherzustellen.

Die Analysen zu den Mobilitätseffekten beruflicher Weiterbildung unterstützen sowohl die Hypothese der Kompensation eines Statusverlusts über Investitionen in berufliche Weiterbildung als auch die Hypothese des nachhaltigen Statuserhalts mittels beruflicher Weiterbildung. Die Chance für nachholende intergenerationale Statusreproduktion durch Weiterbildung ist - der Hypothese der persistenten Ungleichheit nach sozialer Herkunft entsprechend - ebenso wie für den intergenerationalen Statuserhalt im Berufsverlauf nach sozialer Herkunft ungleich verteilt. 
Tabelle 4: $\quad$ Effekte beruflicher Weiterbildung auf intergenerationale Mobilität in Westdeutschland, 1949-1999: Wahrscheinlichkeit eines intergenerationalen Statusabstiegs für statuskonsistente Personen ${ }^{1}$

\begin{tabular}{|c|c|c|c|c|c|c|}
\hline \multirow[t]{2}{*}{ Modell } & \multicolumn{2}{|c|}{1} & \multicolumn{2}{|c|}{2} & \multicolumn{2}{|c|}{3} \\
\hline & $\boldsymbol{\beta}$ & S.E. & $\boldsymbol{\beta}$ & S.E. & $\boldsymbol{\beta}$ & S.E. \\
\hline \multicolumn{7}{|l|}{ Geschlecht } \\
\hline Frau & Referenz & & Referenz & & Referenz & \\
\hline Mann & $-0,598 * * *$ & 0,153 & $-0,552 * * *$ & 0,158 & $-0,519 * * *$ & 0,163 \\
\hline \multicolumn{7}{|l|}{ Zeitdimensionen } \\
\hline Berufserfahrung & $-0,006^{* * *}$ & 0,001 & $-0,007^{* * *}$ & 0,001 & $-0,007^{* * *}$ & 0,001 \\
\hline Arbeitslosenquote & $0,051 * *$ & 0,019 & $0,058 * *$ & 0,020 & $0,050^{*}$ & 0,020 \\
\hline \multicolumn{7}{|l|}{ Aktuelle Klassenlage } \\
\hline Untere Mittelschicht & Referenz & & Referenz & & Referenz & \\
\hline Mittlere Mittelschicht & $-0,909 * * *$ & 0,173 & $-0,803 * * *$ & 0,183 & $-0,687^{* * *}$ & 0,188 \\
\hline Obere Dienstklasse & $-0,619 * * *$ & 0,183 & $-0,317$ & 0,210 & $-0,157$ & 0,216 \\
\hline \multicolumn{7}{|l|}{ Berufliche Weiterbildung } \\
\hline P(Teilnahme) & $-2,379^{*}$ & 1,162 & $-2,264^{*}$ & 1,150 & $-1,887^{+}$ & 1,152 \\
\hline \multicolumn{7}{|l|}{ Schulbildung } \\
\hline Hauptschulabschluss & & & Referenz & & Referenz & \\
\hline Mittlere Reife & & & $-0,101$ & 0,179 & $-0,061$ & 0,183 \\
\hline (Fach)Hochschulreife & & & $-0,740^{*}$ & 0,293 & $-0,616^{*}$ & 0,310 \\
\hline \multicolumn{7}{|l|}{ Berufsausbildung } \\
\hline Kein Abschluss & & & Referenz & & Referenz & \\
\hline Abschluss & & & $-0,528^{+}$ & 0,284 & $-0,471$ & 0,295 \\
\hline \multicolumn{7}{|l|}{ Arbeitsmarktmerkmale } \\
\hline Betriebsgröße & & & & & $-0,071^{*}$ & 0,029 \\
\hline Privatwirtschaft & & & & & Referenz & \\
\hline Öffentlicher Dienst & & & & & $-0,645^{* *}$ & 0,240 \\
\hline Pseudo-R ${ }^{2}$ (McFadden) & 0,0 & & 0,0 & & 0,0 & \\
\hline Episoden / Ereignisse & 4.808 & 23 & 4.808 & 23 & 4.614 & 214 \\
\hline
\end{tabular}

${ }^{1}$ Cox-Regression - nur über 15-jährige Personen in den Mittelschichten in der Zeit ab 1949

${ }^{+} \mathrm{p}=0,1 ;{ }^{*} \mathrm{p}=0,05 ;{ }^{* *} \mathrm{p}=0,01 ;{ }^{* *} \mathrm{p}=0,001$

Datenbasis: GLHS - eigene Berechnungen

\subsection{Diskussion}

Die hier vorgelegten Befunde sprechen dafür, dass der beruflichen Weiterbildung durchaus eine statusreproduzierende Funktion zukommt. Erstens: Intergenerationale Statusabstiege gehen mit Weiterbildungsanstrengungen einher. Obgleich das Statuserhaltmotiv nicht direkt gemessen wurde, wird beobachtet, dass sich intergenerational abgestiegene Erwerbstätige eher weiterbilden als statuskonsistente Personen. Die Neigung, sich nach einem Statusverlust weiterzubilden, ist auch bei Kontrolle individueller Ressourcen wie formaler Erstausbil- dung, beruflicher Stellung, des vorherigen Berufsverlaufs (Berufserfahrung, Tätigkeitswechsel) sowie der institutionellen Restriktionen von Arbeitsmärkten nachweisbar. Somit dürfte es plausibel sein, dass dieses Motiv eine gewichtige subjektive Bedeutung für Weiterbildung und Mobilität hat. Offensichtlich hat die Prägung durch die Klassenlage des Elternhauses als frühzeitigem Sozialisationskontext eine langfristige - mit großen Unterschieden zwischen den sozialen Klassen - im Lebensverlauf nur langsam verblassende Wirkung. 
Zweitens: Intergenerationale Statusverluste können über berufliche Weiterbildung kompensiert werden. Nach einer beruflichen Weiterbildung gelingt es den Teilnehmern eher als den Nichtteilnehmern, über berufliche Aufstiege mindestens wieder in die Klassenlage zu gelangen, die bereits ihre leiblichen oder Stief-Eltern erreicht hatten, als ihre Kinder 15 Jahre alt waren. Drittens: Berufliche Weiterbildung kann möglicherweise - zumindest von beruflich privilegierten Personen - genutzt werden, um Risiken möglicher Statusverluste zu verringern. Diese Befunde sprechen ebenfalls indirekt für die langfristige Wirkung eines im Elternhaus wurzelnden Statuserhaltmotivs.

Nicht nur die Neigung, sich weiterzubilden, ist sozial selektiv, sondern auch die Chancen, die Weiterbildung für die Wiederherstellung und dauerhafte Reproduktion der Statuspositionen erfolgreich nutzen zu können, sind es. Folglich tragen sozial selektive Chancen, sich beruflich weiterbilden und diese erfolgreich nutzen zu können, zur Reproduktion der Klassenstruktur und ungleichen Verteilung von Lebenschancen bei. Denn sowohl die statuskompensierenden als auch die statusreproduzierenden Effekte beruflicher Weiterbildung kommen offensichtlich eher bereits privilegierten Gruppen zugute. Bestehende Ungleichheiten auf den Arbeitsmärkten und in der sozialen Schichtung werden im Sinne des Matthäus-Effektes in der Generationenabfolge reproduziert, verschärfen sich im Lebensverlauf von Geburtskohorten und werden im Aggregat gesehen auf Dauer gestellt. In Bezug auf die soziale Mobilität trägt berufliche Weiterbildung nicht entscheidend zur Eröffnung individueller Lebenschancen für alle bei, sondern eher - wenn auch in der Konsequenz unbeabsichtigt - zur sozialen Schließung in der gesellschaftlichen Sozial- und Klassenstruktur zugunsten privilegierter Sozialgruppen.

\section{Fazit}

Im vorliegenden Beitrag wurden Mobilitätseffekte beruflicher Weiterbildung unter folgender Fragestellung untersucht: Erfolgen Teilnahmen an beruflicher Weiterbildung unter anderem mit dem Ziel, etwaige - gemessen an der Klassenlage des Elternhauses - Statusverluste im Bildungs- und Berufsverlauf zu kompensieren? Falls ja: Ist die berufliche Weiterbildung ein geeignetes Mittel, um intergenerationale Abwärtsmobilität wettzumachen oder um Risiken für intergenerationale Abstiege zu minimieren? Aus der Sicht der strukturell-individualistischen Erklärung ist diese empirisch zu überprü- fende Fragestellung ein Test für die in der soziologischen Bildungsforschung betonte, aber auch umstrittene Rolle des Statuserhaltmotivs bei Bildungs- und Berufsentscheidungen, weil zum einen über einen längeren Zeitraum des Lebensverlaufs die nachhaltige Wirkung einer möglicherweise durch das Elternhaus angestrebten Statusreproduktion beobachtet wird. Zum anderen liegt empirische Evidenz vor, wonach bei gegebenem Statuserhaltmotiv der intra- und intergenerationalen Klassenmobilität in späteren Berufsverlaufsphasen qualifikatorische und strukturelle Restriktionen der Arbeitsmärkte entgegenstehen.

Diese Fragestellung wurde mit ereignisorientierten Verlaufsdaten der Deutschen Lebensverlaufsstudie für westdeutsche Frauen und Männer in unterschiedlichen Geburtskohorten für die historische Periode von 1949 bis 1999 untersucht. Die Annahmen zur Teilnahme an und zur Auswirkung von beruflicher Weiterbildung werden weitestgehend empirisch untermauert. Intergenerational abgestiegene Erwerbstätige bilden sich eher weiter als statuskonsistente Personen. In der Regel können sie mittels beruflicher Weiterbildung ihre Statusverluste ausgleichen, während statuskonsistente Erwerbstätige sie erfolgreich für den Statuserhalt nutzen können.

Abschließend soll nochmals auf Defizite der vorliegenden Analyse aufmerksam gemacht werden, die mit informationsreicheren Daten (etwa aus dem Nationalen Bildungspanel) behoben werden sollten. Zum einen wurde das individuelle Statuserhaltmotiv nicht direkt gemessen, sondern theoriegeleitet aus dem beobachteten Weiterbildungsverhalten und den Effekten der Weiterbildungsteilnahme für bestimmte Risikomengen geschlossen. Deswegen kann nicht ausgeschlossen werden, dass ganz andere Motive zur Weiterbildung einschließlich ihrer Konsequenzen für den Berufsverlauf ausschlaggebend waren. Das Statuserhaltmotiv theoretisch konsistent und gleichzeitig real zufällig mit dem intergenerationalen Statusabstieg korrelieren. In der Wirkung ist auch nicht ausgeschlossen, dass gänzlich andere Absichten, sich beruflich weiterzubilden - wie etwa Verbesserung der Chancen für eine Beförderung oder das Streben nach Einkommensgewinnen - zur Statusreproduktion führen. Wahrscheinlich deswegen ist die statistische Erklärungskraft unserer Modellierungen vergleichsweise gering. Daher sollte die Bedeutung von beruflicher Weiterbildung für die intergenerationale Statusreproduktion im Vergleich zum erfolgreichen statuserhaltenden Berufseinstieg und zur statuskonservierenden beruflichen Karriere (etwa infolge von Seniorität) nicht überschätzt werden. Zum anderen 
konnte die strukturelle Heterogenität der besuchten Weiterbildungskurse, die Dauer und die Häufigkeit von Weiterbildungsteilnahmen nicht kontrolliert werden. Gründe hierfür waren sowohl fehlende Fragen bei der Datenerhebung als auch fehlende bzw. ungenaue Angaben der Befragten in älteren Geburtskohorten. In diesem Sinne sollten unsere Ergebnisse zu weiterführenden Replikationen anregen.

\section{Literatur}

Backes-Gellner, U., J. Mure \& S.N. Tuor, 2007: The Puzzle of Non-Participation in Continuing Training An Empirical Study of Chronic vs. Temporary NonParticipation. Zeitschrift für Arbeitsmarktforschung 2: 295-311.

Bardeleben, R., U. Beicht \& J. Holzschuh, 1994: Individuelle Kosten und Nutzen der beruflichen Weiterbildung. BWP 23: 9-17.

Becker, R., 1991: Berufliche Weiterbildung und Berufsverlauf. Mitteilungen aus der Arbeitsmarkt- und Berufsforschung 24: 351-364.

Becker, R., 1994: Intergenerationale Mobilität im Lebensverlauf oder: Ist der öffentliche Dienst ein Mobilitätskanal zwischen Generationen? Kölner Zeitschrift für Soziologie und Sozialpsychologie 46: 596-617.

Becker, R. \& K. Schömann, 1996: Berufliche Weiterbildung und Einkommensdynamik. Kölner Zeitschrift für Soziologie und Sozialpsychologie 48: 426-461.

Becker, R., S. Haunberger \& F. Schubert, 2010: Studienfachwahl als Spezialfall der Ausbildungsentscheidung und Berufswahl. Zeitschrift für Arbeitsmarktforschung 42: 292-310.

Becker, R. \& A.E. Hecken, 2007: Studium oder Berufsausbildung? Zeitschrift für Soziologie 36: 100-117.

Becker, R. \& A.E. Hecken, 2011: Berufliche Weiterbildung - bildungs- und arbeitsmarktsoziologische Perspektiven und empirische Befunde. S. 367-410 in: R. Becker (Hrsg.), Lehrbuch der Bildungssoziologie. Wiesbaden: VS.

Beicht, U. \& G. Walden, 2006: Individuelle Investitionen in berufliche Weiterbildung - Heutiger Stand und künftige Anforderungen. WSI-Mitteilungen 59: 327-334.

Blossfeld, H.-P., 1989: Kohortendifferenzierung und Karriereprozeß. Frankfurt a.M.: Campus.

Blossfeld, H.-P. \& G. Rohwer, 1995: Techniques of Event History Modeling. Mawah: Erlbaum.

Blossfeld, H.-P. \& G. Rohwer, 1997: Causal Inference, Time and Observation Plans in the Social Sciences. Quality \& Quantity 31: 364-384.

Blossfeld, H.-P. \& J. Huinink, 2001: Lebensverlaufsforschung als sozialwissenschaftliche Forschungsperspektive. BIOS 14: 5-31.

Böhnke, P., 1997: Zum Stellenwert beruflicher Weiterbildung in den ersten Jahren des Erwerbsverlaufs. Theoretische Überlegungen und empirische Befunde zur be- ruflichen Weiterqualifizierung der Geburtsjahrgänge 1954-56 und 1959-61. Diplomarbeit. Berlin: Freie Universität Berlin.

Boudon, R., 1974: Education, Opportunity, and Social Inequality. New York: Wiley.

Breen, R. \& M. Yaish, 2006: Testing the Breen-Goldthorpe Model of Educational Decision Making. S. 232-258 in: S.L. Morgan, D.B. Grusky \& G.S. Fields (Hrsg.), Mobility and Inequality. Stanford: Stanford University Press.

Brückner, E. \& K.U. Mayer, 1998: Collecting Life History Data: Experiences from the German Life History Study. S. 152-183 in: J.Z. Giele \& G.H. Elder (Hrsg.), Methods of Life Course Research. Qualitative and Quantitative Approaches. Thousand Oaks: Sage.

Brüderl, J., 2004: Die Überprüfung von Rational-ChoiceModellen mit Umfragedaten. S. 163-180 in: A. Diekmann \& T. Voss (Hrsg.): Rational-Choice-Theorie in den Sozialwissenschaften. München: Oldenbourg.

Büchel, F. \& M. Pannenberg, 2004: Berufliche Weiterbildung in West- und Ostdeutschland. Teilnehmer, Struktur und individuelle Förderung. Zeitschrift für Arbeitsmarktforschung 2: 73-126.

Eisermann, M., F. Janik \& T. Kruppe, 2014: Weiterbildungsbeteiligung - Ursachen unterschiedlicher Teilnahmequoten in verschiedenen Datenquellen. Zeitschrift für Erziehungswissenschaft 17: 473-495.

Gabay-Egozi, L., Y. Shavit \& M. Yaish, 2009: Curricular Choice: A Test of a Rational Choice Model of Education. European Sociological Review 26: 447-463.

Glauser, David, 2015: Berufsausbildung oder Allgemeinbildung? Ungleichheiten nach sozialer Herkunft, Geschlecht und Migrationshintergrund beim Übergang in Ausbildung der Sekundarstufe II. Wiesbaden: Springer.

Granovetter, M., 1974: Getting a Job. Cambridge, Mass.: Harvard University Press.

Haller, A.O. \& A. Portes, 1973: Status Attainment Processes. Sociology of Education 46: 51-91.

Heckman, J.J., 1979: Sample Selection Bias as a Specification Error. Econometrica 47: 153-161.

Hillmert, S., 2004: Die Westdeutsche Lebensverlaufsstudie, Kohorten 1964 und 1971: Projekt, Datenerhebung und Edition. S. 215-230 in: S. Hillmert \& K.U. Mayer (Hrsg.), Geboren 1964 und 1971. Wiesbaden: VS.

Jacob, M. \& F. Weiss, 2011: Class Origin and Young Adults` Re-enrollment. Research in Social Stratification and Mobility 29: 415-426.

Jæger, M.M., \& A. Holm, 2012: Conformists or Rebels? Relative Risk Aversion, Educational Decisions and Social Class Reproduction. Rationality \& Society 24: 221-253.

Kahneman, D. \& A. Tversky, 1979: Prospect Theory. An Analysis of Decision under Risk. Econometrica 39: 342-350.

Keller, S. \& M. Zavalloni, 1964: Ambition and Social Class: A Respecification. Social Forces 43: 58-70.

Kuwan, H. \& F. Thebis, 2005: Berichtssystem Weiterbildung 2003. Integrierter Gesamtbericht zur Weiterbildungssituation in Deutschland (Herausgegeben vom 
Bundesministerium für Bildung und Forschung). Bonn: $\mathrm{BMBF}$

Matthes, B., M. Reimer \& R. Künster, 2007: Techniken und Werkzeuge zur Unterstützung der Erinnerungsarbeit bei der computergestützten Erhebung retrospektiver Längsschnittdaten. Methoden - Daten - Analysen 1: 69-92.

Mayer, K.U., 2008: Retrospective Longitudinal Research: The German Life History Study. S. 85-106 in: S.W. Menard (Hrsg.), Handbook of Longitudinal Research: Design, Measurement and Analysis. San Diego: Elsevier.

Mayer, K.U. \& G.R. Carroll, 1987: Jobs and Classes: Structural Constraints on Career mobility. European Sociological Review 3: 14-38.

Mayer, K.U. \& H.-P. Blossfeld, 1990: Die gesellschaftliche Konstruktion sozialer Ungleichheit im Lebenslauf. S. 297-318 in: P.A. Berger \& S. Hradil (Hrsg.), Lebenslagen - Lebensläufe - Lebensstile (Sonderband 7 der Sozialen Welt). Göttingen: Schwartz.

Mayer, K.U. \& J. Huinink, 1990: Alters-, Perioden- und Kohorteneffekte in der Analyse von Lebensverläufen oder: Lexis ade? S. 442-460 in: K.U. Mayer (Hrsg.), Lebensverläufe und sozialer Wandel. Opladen: Westdeutscher Verlag.

Mayer, K.U. \& H. Solga, 1994: Mobilität und Legitimität. Zum Vergleich der Chancenstrukturen in der alten DDR und der alten BRD oder: Haben Mobilitätschancen zu Stabilität und Zusammenbruch der DDR beigetragen? Kölner Zeitschrift für Soziologie und Sozialpsychologie 46: 193-208.

Mayer, K.U. \& S. Aisenbrey, 2007: Variations on a Theme: Trends in Social Mobility in (West) Germany for Cohorts Born between 1919 and 1971. S. 125-154 in: S. Scherer, R. Pollak, G. Otte \& M. Gangl (Hrsg.), From Origin to Destination. Frankfurt a.M.: Campus.

Morgan, S.L. \& C. Winship, 2007: Counterfactuals and Causal Inference. Cambridge: Cambridge University Press.

Müller, W. \& R. Pollak, 2004: Social Mobility in West Germany. The Long Arms of History Discovered? S. 77-113 in: R. Breen (Hrsg.), Social Mobility in Europe. Oxford: Oxford University Press.

Müller, W. \& M. Jacob, 2008: Qualifications and the Returns to Training Across the Life Course. S. 126-172 in: K.U. Mayer \& H. Solga (Hrsg.), Skill Formation. Interdisciplinary and Cross-National Perspectives. Cambrige: Cambridge University Press.

Offerhaus, J., J. Leschke \& K. Schömann, 2010: Soziale Ungleichheit im Zugang zu beruflicher Weiterbildung. S. 345-375 in: R. Becker \& W. Lauterbach (Hrsg.), Bildung als Privileg. Wiesbaden: VS.
Ormel, J., S. Lindenberg, N. Steverink \& L.M. Verbrugge, 1999: Subjective Well-being and Social Production Functions. Social Indicator Research 46: 61-90.

Pötter, U. \& H.-P. Blossfeld, 2001: Causal Inference from Series of Events. European Sociological Review 17: 21-32.

Reimer, M., 2003: Autobiografisches Gedächtnis und retrospektive Datenerhebung: die Rekonstruktion und Validität von Lebensverläufen. Dissertation. Berlin: Freie Universität Berlin.

Reimer, D. \& R. Pollak, 2010: Educational Expansion and Its Consequences for Vertical and Horizontal Inequalities in Access to Higher Education in West Germany. European Sociological Review 26: 415-430.

Schindler, S. \& D. Reimer, 2010: Primäre und sekundäre Effekte der sozialen Herkunft beim Übergang in die Hochschulbildung. Kölner Zeitschrift für Soziologie und Sozialpsychologie 62: 623-653.

Schindler, S., F. Weiss \& T. Hubert, 2011: Explaining the Class Gap in Training: The Role of Employment Relations and Job Characteristics. International Journal of Lifelong Learning 30: 213-232.

Schömann, K. \& R. Becker, 1995: Participation in Further Education over the Life Course. European Sociological Review 11: 187-208.

Sewell, W.H., A.O. Haller \& A. Portes, 1969: The Educational and Early Occupational Attainment Process. American Sociological Review 34: 82-92.

Stocké, V., 2007: Explaining Educational Decision and Effects of Families Social Class Position: An Empirical Test of the Breen-Goldthorpe Model of Educational Attainment. European Sociological Review 23: 505519.

Stocké, V., 2010: Der Beitrag der Theorie rationaler Entscheidung zur Erklärung von Bildungsungleichheit und Bildungsarmut. S. 73-95 in: G. Quenzel \& K- Hurrelmann (Hrsg.), Bildungsverlierer. Neue Ungleichheiten. Wiesbaden: VS.

Van de Werfhorst, H.G. \& S. Hofstede, 2007: Cultural Capital or Relative Risk Aversion? Two Mechanisms for Educational Inequality Compared. British Journal of Sociology 58: 391-415.

Wegener, B., 1989: Kritik des Prestige. Opladen: Westdeutscher Verlag.

Winship, C. \& M. Sobel, 2004: Causal Inferences in Sociological Studies. S. 481-503 in: M. Hardy \& A. Bryman (Hrsg.), Handbook of Data Analysis. London: Sage.

Wolter, F. \& J. Schiener, 2009: Einkommenseffekte beruflicher Weiterbildung. Empirische Analysen auf Basis des Mikrozensus-Panels. Kölner Zeitschrift für Soziologie und Sozialpsychologie 61: 90-117. 


\section{Autorenvorstellung}

Rolf Becker, geb. 1960 in Dillingen (Saar). Studium der Soziologie, Sozialpsychologie, Politikwissenschaft und Zeitgeschichte in Mannheim. Promotion in Berlin. Habilitation in Dresden. Seit 2004 Professor für Bildungssoziologie in Bern.

Forschungsschwerpunkte: Bildungssoziologie, Sozialstrukturanalyse, Lebensverlaufsforschung, Methoden der empirischen Sozialforschung und angewandte Statistik, Arbeitsmarkt- und Mobilitätsforschung.

Jüngste Publikationen: Die Bildungsexpansion in der Schweiz und ihre Folgen. Eine empirische Analyse des Wandels der Bildungsbeteiligung und -ungleichheiten mit den Daten der Schweizer Volkszählungen 1970, 1980, 1990 und 2000 (mit C. Zangger), in: Kölner Zeitschrift für Soziologie und Sozialpsychologie 2013; Reversal of Gender Differences in Educational Attainment - Historical Analysis of the West German Case, in: Educational Research 2014; zuletzt in dieser Zeitschrift: Wirkungen monetärer Anreize auf Antworten zur eigenen Delinquenz bei postalischer Befragung (mit G. Mehlkop), ZfS 44 (3), 2015: 215-228.

Klaus Schömann, geb. 1961 in Wittlich. Studium der Volkswirtschaftslehre und Politikwissenschaft in Saarbrücken (Deutschland) und Cardiff (Wales). Promotion an der FU Berlin. 2004-2014 Professor für Soziologie an der Jacobs University in Bremen. Seit 2014 Professor und Programmleiter am DIE (Deutsches Institut für Erwachsenenbildung, Leibniz-Zentrum für Lebenslanges Lernen e.V.) in Bonn.

Forschungsschwerpunkte: Lebensverlaufsforschung, lebenslanges Lernen und alternde Gesellschaften, Arbeitsmarktund Berufsforschung.

Jüngste Publikationen: Different Contexts, Different Effects? Work Time and Mental Health in the United States and Germany (mit S. Kleiner \& R. Schunck), in: Journal of Health and Social Behavior 2015 (im Erscheinen); Der Beitrag von Wohlfahrtsstaatsregimen und Varianten kapitalistischer Wirtschaftssysteme zur Erklärung von Weiterbildungsteilnahmestrukturen bei Ländervergleichen (mit K. Kaufmann \& E. Reichart), in: Zeitschrift für Weiterbildungsforschung 2014; zuletzt in dieser Zeitschrift: Erwerbsverlauf und die Entwicklung der Arbeitseinkommen bei Männern - Eine Längsschnittanalyse unter Verwendung einer stochastischen Differentialgleichung (mit H.-P. Blossfeld \& M.T. Hannan), ZfS 17 (6), 1988: 407-423. 